\title{
Induction of synergistic non-apoptotic cell death by simultaneously targeting proteasomes with bortezomib and histone deacetylase 6 with ricolinostat in head and neck tumor cells
}

\author{
KAZUHIRO HATTORI ${ }^{1}$, NAOHARU TAKANO ${ }^{2}$, HIROMI KAZAMA ${ }^{2}$, SHOTA MORIYA ${ }^{2}$, \\ KEITARO MIYAKE ${ }^{1}$, MASAKI HIRAMOTO ${ }^{2}$, KIYOAKI TSUKAHARA ${ }^{1}$ and KEISUKE MIYAZAWA ${ }^{2}$ \\ Departments of ${ }^{1}$ Otolaryngology, Head and Neck Surgery and ${ }^{2}$ Biochemistry, \\ Tokyo Medical University, Tokyo 160-8402, Japan
}

Received April 2, 2021; Accepted June 25, 2021

DOI: $10.3892 / \mathrm{ol} .2021 .12941$

\begin{abstract}
Following surgery and chemoradiation, $50 \%$ of patients with locally advanced head and neck tumors experience relapse within the firsttwoyears, with apoor prognosis. Therefore, a novel therapeutic approach is required. The aim of the present study was to investigate the effect of combination treatment with the proteasome inhibitor bortezomib (BTZ), and ricolinostat (RCS), a specific inhibitor of histone deacetylase 6 (HDAC6), on CAL27 and Detroit562 head and neck cancer cells. BTZ and RCS exhibited cytotoxicity in a dose- and time-dependent manner. Simultaneous treatment with BTZ and RCS resulted in the synergistic enhancement of non-apoptotic cell death and autophagy. The receptor-interacting serine/threonine-protein kinase 1 (RIPK1) inhibitor, necrostatin, but not the autophagy inhibitor, 3-methyladenine, attenuated the cytotoxicity of combined BTZ and RCS treatment. Thus, necroptosis [type-III programmed cell death (PCD)], but not autophagic cell death (type-II PCD), appeared to contribute to the pronounced cytotoxicity. However, no phosphorylation of RIPK1 or mixed lineage kinase domain-like protein was detectable in response to BTZ or RCS. Furthermore, RCS induced $\alpha$-tubulin acetylation and inhibited BTZ-induced aggresome formation along with endoplasmic reticulum stress loading. Combined treatment with BTZ and RCS enhanced the production of reactive oxygen species (ROS). The ROS scavenger, $\mathrm{N}$-acetyl cysteine, abrogated the increase in cytotoxicity. These results
\end{abstract}

Correspondence to: Dr Naoharu Takano or Professor Keisuke Miyazawa, Department of Biochemistry, Tokyo Medical University, 6-1-1 Shinjuku, Shinjuku-ku, Tokyo 160-8402, Japan

E-mail: ntakano@tokyo-med.ac.jp

E-mail:miyazawa@tokyo-med.ac.jp

Key words: ricolinostat, histone deacetylase 6, bortezomib, proteasome, necroptosis, head and neck tumor, squamous cell carcinoma suggest the potential therapeutic value of the dual targeting of the proteasome and HDCA6 for head and neck cancers through the induction of necroptosis-like cell death along with ROS generation.

\section{Introduction}

Worldwide, head and neck cancers account for $>650,000$ cases and 330,000 deaths annually (1). Surgery and chemoradiation are the primary treatments for head and neck cancer, and in previous years, therapeutic options have increased with the development of molecular targeting drugs and immune checkpoint inhibitors (2). However, $50 \%$ of patients with locally advanced tumors still relapse within the first two years after treatment for recurrent or metastatic disease. Once relapsed, the response rates for chemoradiation range from 10 to $35 \%$, with a median survival time of $6-12$ months $(1,2)$. Therefore, the development of novel therapies is urgently required.

Histone deacetylase 6 (HDAC6) is a zinc-dependent member of the class IIb HDAC family, which structurally differs from the other family members due to its dual deacetylase domains, as well as a ubiquitin-binding domain $(3,4)$. Unlike other HDACs, HDAC6 functions as a cytoskeletal-modulating enzyme through the deacetylation of $\alpha$-tubulin, and also binds to ubiquitinated misfolded protein complexes for degradation via the ubiquitin-proteasome system (UPS) (4-6). HDAC6 regulates the molecular chaperone activity of heat shock protein 90 (HSP90) through deacetylation (7). In response to the accumulation of misfolded protein aggregates, HDAC6 dissociates from the HSP90 chaperone complex to bind ubiquitinated protein aggregates and delivers them to the proteasome $(6,8,9)$. Additionally, HDAC6 promotes autophagy by recruiting cortactin-dependent actin-remodeling machinery, which in turn assembles an F-actin network that stimulates autophagosome-lysosome fusion, leading to substrate degradation $(8,10)$. Thus, HDAC6 is believed to play a crucial role in maintaining cellular homeostasis by aiding the protein chaperone network to fold misfolded proteins, as well as clearing misfolded aggregates through the UPS and autophagy-lysosome system $(8,9)$. 
Cytoplasmic accumulation of misfolded proteins contributes to the pathogenesis of multiple diseases, including cancer (10). In breast cancer cells, combined treatment with the proteasome inhibitor bortezomib (BTZ) and either a specific inhibitor of HDAC6 (tubacin) or vorinostat [suberoylanilide hydroxamic acid (SAHA)], a pan-HDAC inhibitor that potently inhibits HDAC6, induces pronounced apoptosis via endoplasmic reticulum (ER) stress loading (11).

Ricolinostat (RCS; drug development code ACY-1215) is the first orally bioavailable HDAC6 inhibitor with potential anticancer activity, as demonstrated in patients with myeloma and a xenograft mouse model of diffuse large cell lymphoma $(8,12)$. RCS selectively binds HDAC6, disrupts the HSP90 protein chaperone system through the hyperacetylation of HSP90, and prevents subsequent aggresomal protein degradation in leukemia and myeloma cell lines $(8,9,13)$.

Simultaneous targeting of the proteasome and HDAC6 potently induces apoptosis in myeloma cells via ER stress loading (13). Our previous studies reported that dual targeting of proteasome with BTZ, and autophagy with macrolide antibiotics (which inhibit autophagy as an off-target effect), enhanced apoptosis and ER stress loading in myeloma and breast cancer cells $(14,15)$. Moreover, a real-time quantitative ER stress monitoring system was used to demonstrate that the simultaneous inhibition of proteasomes with BTZ, autophagy with clarithromycin, and aggresome formation with SAHA resulted in the most prominent ER stress loading and apoptosis induction, compared with the effects of any combination of two reagents in a breast cancer cell line (16). These data strongly suggest the existence of an intracellular mutual network comprising the proteasome, autophagy and aggresome to maintain cellular homeostasis against excess ER stress loading. Thus, targeting these comprehensive cellular processing systems for misfolded proteins, namely ubiquitin-proteasome system, autophagy, and aggresome formation, appears to be a novel strategy for cancer therapy $(15,16)$.

Most previous studies targeting intracellular protein processing have been conducted in adenocarcinoma and myeloma, which are characterized by potent protein secretion $(10,11,13-16)$. To the best of our knowledge, there are no studies published addressing squamous cell carcinoma, including head and neck tumors, in this context. The aim of the present study was to investigate the therapeutic potential of combined BTZ and RCS treatment for head and neck tumors.

\section{Materials and methods}

Reagents. BTZ and RCS were purchased from Selleck Chemicals, and were dissolved in dimethyl sulfoxide (DMSO; Nacalai Tesque, Inc.) to generate stock solutions (BTZ, $1 \mathrm{mM}$; RCS, $10 \mathrm{mM}$ ). Z-VAD-fmk, a pan-caspase inhibitor, was purchased from Peptide Institute, Inc. 3-Methyladenine (3-MA) was obtained from Tokyo Chemical Industry Co., Ltd. Necrostatin-1, a specific inhibitor of receptor-interacting serine/threonine-protein kinase 1 (RIPK1), was purchased from Enzo Life Sciences, Inc. Cycloheximide (CHX) was purchased from Calbiochem. N-acetyl-L-cysteine (NAC). Bafilomycin $\mathrm{A}_{1}$, rapamycin, puromycin dihydrochloride, recombinant human TNF- $\alpha$ and staurosporine were purchased from FUJIFILM Wako Pure Chemical. Thapsigargin (TPG) was purchased from Nacalai Tesque, Inc.

Cell lines and culture conditions. The CAL27 human oral squamous cell carcinoma cell line and Detroit562 human pharyngeal squamous carcinoma cell line were obtained from the American Type Culture Collection (ATCC). All cell lines were cultured in Dulbecco's modified Eagle's medium (DMEM; Sigma-Aldrich; Merck KGaA) containing 10\% fetal bovine serum (Gibco; Thermo Fisher Scientific, Inc.) and $1 \%$ penicillin/streptomycin solution (FUJIFILM Wako Pure Chemical) in a humidified incubator $\left(37^{\circ} \mathrm{C}, 5 \% \mathrm{CO}_{2}\right.$ and $95 \%$ air). All cell line experiments were conducted within 10 passages after thawing. Mycoplasma contamination was tested routinely using the e-Myco ${ }^{\text {TM }}$ Mycoplasma PCR Detection kit ver. 2.0 (Intron Biotechnology, Inc.) according to the manufacturer's instructions. As a positive control for necroptosis induction, HT-29 cells (ATCC), a colorectal adenocarcinoma cell line, were authenticated using STR profiling, pre-treated with Z-VAD-fmk $(20 \mu \mathrm{M})$ in DMEM for $30 \mathrm{~min}$, followed by additional treatment with CHX $(10 \mu \mathrm{g} / \mathrm{ml})$ and human TNF- $\alpha$ (20 ng/ml) for $8 \mathrm{~h}$, as described previously (17).

Assessment of cellular proliferation inhibition. Cellular proliferation was assessed using the CellTiter Blue Cell Viability Assay kit (Promega Corporation) according to the manufacturer's instructions. Briefly, cells were seeded into a 96-well flat-bottom culture plate $\left(3 \times 10^{3}\right.$ cells/well) and pre-cultured for $24 \mathrm{~h}$. Once fully adhered to the plate, the medium was replaced, and the cells were treated with BTZ (2.5-10 nM) and RCS (1-10- $\mu \mathrm{M})$ for 24 or $48 \mathrm{~h}$. All untreated controls were supplemented with DMSO to match the volume of BTZ and/or RCS treatments. During the last $1.5 \mathrm{~h}$, CellTiter Blue reagent was added to each well, and fluorescence was measured (excitation, $560 \mathrm{~nm}$; emission, $590 \mathrm{~nm}$ ) using a SpectraMax iD3 fluorometer (Molecular Devices, LLC). The mean fluorescence relative to that of the untreated cells was expressed as a percentage of cellular proliferation.

Morphological assessment. Cells were seeded onto a 60-mm dish $\left(1 \times 10^{6}\right.$ cells/dish) and pre-cultured for $24 \mathrm{~h}$. After treatment with BTZ and/or RCS for $48 \mathrm{~h}$, adherent cells were harvested by trypsinization. The cells were spread on glass slides by centrifugation $(1,000 \mathrm{x} \mathrm{g}$ for $5 \mathrm{~min}$ at room temperature, then stained with May-Grünwald-Giemsa (Muto Pure Chemicals Co., Ltd.), as previously described (17). Samples were examined by digital light microscopy using a BZ-X810 microscope (Keyence Corporation).

Flow cytometry. To assess apoptosis, $1 \times 10^{6}$ cells $/ \mathrm{ml}$ cells were resuspended in annexin $\mathrm{V}$ binding buffer and stained using the Annexin V-FITC Apoptosis Detection kit (Nacalai Tesque, Inc.) according to the manufacturer's instructions. Flow cytometry was performed using an Attune Acoustic Focusing Cytometer, and data analysis was performed using Attune Cytometer software v. 2.1.0 (both Thermo Fisher Scientific, Inc.).

Immunoblotting. Immunoblotting was performed as previously described (18). Briefly, cells were seeded onto a 60-mm dish 
( $1 \times 10^{6}$ cells/dish), and pre-cultured for $24 \mathrm{~h}$. The cells were then treated as aforementioned for the optimal duration, then lysed with RIPA buffer (cat. no. 08714-04; Nacalai Tesque, Inc.) supplemented with a protease and phosphatase inhibitor cocktail (cat. no. 07574-61; Nacalai Tesque, Inc.). Cellular proteins were quantified using the Pierce ${ }^{\mathrm{TM}}$ BCA Protein Assay kit (Thermo Fisher Scientific, Inc.) according to the manufacturer's instructions. Proteins $(15 \mu \mathrm{g})$ were loaded and separated by sodium dodecyl sulfate-polyacrylamide gel electrophoresis (SDS-PAGE) (7.5, 10 and 15\% gels were used) and transferred to Immobilon-P membranes (MilliporeSigma). After that, the membrane was blocked with $3 \%$ skim milk or $3 \%$ BSA (for phospho-proteins) for $1 \mathrm{~h}$ at RT.-BLUE Star PLUS Prestained Protein-Ladder (cat. no. NE-MWP04; Nippon Genetics Co., Ltd.) was loaded onto the gel as a protein molecular weight marker.

The membranes were probed with the following primary antibodies (Abs): i) Anti-microtubule-associated protein 1 light chain 3 (LC3) B (cat. no. NB600-1384; 1:4,000; Novus Biologicals, Ltd.); anti-caspase-3 (cat. no. 9662S; 1:1,000), anti-phospho-RIPK1 (Ser166; cat. no. 65746S; $1: 1,000)$ and anti-C/EBP homologous protein (CHOP; cat. no. 2895S; 1:1,000) (all from Cell Signaling Technology, Inc.); ii) anti-glucose-regulated protein of $78 \mathrm{kDa}$ (GRP78; cat. no. sc-13968), anti-ubiquitin monoclonal Ab (mAb) (cat. no. sc-8017), anti- $\alpha$-tubulin mAb (cat. no. sc-5286), anti-acetylated- $\alpha$-tubulin mAb (cat. no. sc-23950), anti-p62 (sequestosome-1) mAb (cat. no. sc-28359) and anti- $\beta$-actin mAb (cat. no. sc-47778) (all 1:1,000; Santa Cruz Biotechnology, Inc.); and iii) anti-mixed lineage kinase domain-like protein (MLKL; cat. no. ab183770) and anti-phospho-MLKL (Ser 358; cat. no. ab187091) (both from 1:1,000; Abcam) for $16 \mathrm{~h}$ at $4^{\circ} \mathrm{C}$. Immunoreactive proteins were detected with horseradish peroxidase-conjugated secondary Abs (either anti-mouse; cat. no. 115-035-003; 1:2,500; or anti-rabbit; cat. no. 711-035-152; 1:2,500; both from Jackson ImmunoResearch) for $1 \mathrm{~h}$ at room temperature. Immobilon Western Chemiluminescent HRP Substrate (cat. no. WBKLS0500; Merck Millipore) was added and densitometry was performed using a WSE-6300H/C Luminograph III (ATTO) and CSAnalyzer4 software version 2.3.1 (ATTO).

Immunofluorescence staining and confocal microscopy. CAL27 and Detroit562 cells were seeded onto 13-mm glass coverslips in a 24 -well culture plate $\left(1 \times 10^{5}\right.$ cells/well), and pre-cultured for $24 \mathrm{~h}$. After treatment with BTZ and/or RCS for $24 \mathrm{~h}$, coverslips were washed twice with PBS and fixed for $10 \mathrm{~min}$ in $2 \%$ paraformaldehyde in PBS at room temperature. After washing with PBS, the cells were permeabilized with $0.1 \%$ Triton X-100 in TBST for $5 \mathrm{~min}$ at room temperature. The coverslips were then washed with TBST and further incubated with TBST containing $10 \%$ newborn calf serum (NCS; Thermo Fisher Scientific, Inc.) for $60 \mathrm{~min}$ at room temperature to block nonspecific binding. The cells were then incubated with mouse anti-ubiquitin (cat. no. sc-8017; 1:100), keratin-17 (cat. no. sc-393002; 1:1,000) and acetylated $\alpha$-tubulin mAb (cat. no. 23950; 1:100) (all from Santa Cruz Biotechnology, Inc.) diluted in TBST containing $1.5 \%$ NCS and $0.1 \%$ bovine serum albumin (BSA; FUJIFILM Wako
Pure Chemical Corporation) at $4^{\circ} \mathrm{C}$ overnight. The coverslips were washed with TBST at room temperature (three times for 5 min each) and subsequently incubated with Alexa Fluor 488 goat anti-mouse IgG $(\mathrm{H}+\mathrm{L})$ secondary Ab (Thermo Fisher Scientific, Inc.; cat. no. A11029; 1:1,000) in TBST containing $1.5 \% \mathrm{NCS}$ and $0.1 \% \mathrm{BSA}$, for $60 \mathrm{~min}$ at $37^{\circ} \mathrm{C}$. The coverslips were washed with TBST and mounted using ProLong Diamond Antifade Montant (Thermo Fisher Scientific, Inc.). Nuclei were stained with DAPI. The cells were imaged by confocal laser scanning fluorescence microscopy using an LSM 700 microscope (Zeiss AG) and analyzed using ZEN 2.3 SP1 Black Edition software (Zeiss AG).

Assessment of aggresome by fractioning detergent-soluble and detergent-insoluble proteins followed by immunoblotting with anti-ubiquitin $m A b$. CAL27 cells $\left(1 \times 10^{6}\right)$ were seeded in a $60-\mathrm{mm}$ dish and pre-cultured for $24 \mathrm{~h}$. The cells were then treated with BTZ \pm RCS for 24 or $48 \mathrm{~h}$, then lysed with Triton X-100 lysis buffer (10 mM Tris- $\mathrm{HCl}, 150 \mathrm{mM} \mathrm{NaCl}$ and $2 \%$ Triton $\mathrm{X}-100, \mathrm{pH} 7.8$ ) supplemented with a protease inhibitor cocktail (Nacalai Tesque, Inc.). The lysates were centrifuged at $12,000 \mathrm{x}$ g for $30 \mathrm{~min}$ at $4^{\circ} \mathrm{C}$, after which the supernatant was collected as a detergent-soluble fraction. The pellets containing insoluble proteins were individually resuspended in sodium dodecyl sulfate (SDS) lysis buffer $(10 \mathrm{mM}$ Tris-HCl; $150 \mathrm{mM} \mathrm{NaCl} ; 2 \%$ SDS, $\mathrm{pH} 7.8$ ) and sonicated for 30 sec using a VP-5S tip sonicator (TAITEC Corporation) to prepare a detergent-insoluble fraction. All resuspended pellets and supernatants were boiled for $5 \mathrm{~min}$ in the presence of an equal volume of SDS-PAGE sample buffer (125 mM Tris-HCl; 4\% SDS; $20 \%$ glycerol; $0.002 \%$ bromophenol blue, $\mathrm{pH} 6.8$ ). The proteins were separated using 5-20\% SDS-PAGE gels and immunoblotted with anti-ubiquitin mAb (cat. no. sc-8017; 1:1,000; Santa Cruz Biotechnology, Inc.) as described in the immunoblotting section. After detection, the membrane was stained with $0.25 \%$ Coomassie Brilliant Blue R-250 (FUJIFILM Wako Pure Chemical Corporation) for $1 \mathrm{~h}$ at room temperature to compare the amount protein loaded.

Transmission electron microscopy (TEM). TEM analyses were performed at the Hanaichi Ultrastructure Research Institute (Aichi, Japan). CAL27 cells $\left(1 \times 10^{6}\right)$ were seeded into a 60-mm dish and pre-cultured for $24 \mathrm{~h}$. Cells were treated with or without BTZ (5 nM) and/or RCS $(5 \mu \mathrm{M})$ for $24 \mathrm{~h}$, and then fixed with $2.5 \%$ glutaraldehyde in $0.1 \mathrm{M}$ phosphate buffer (pH 7.4) for $16 \mathrm{~h}$ at $4^{\circ} \mathrm{C}$. The samples were further fixed in $2 \%$ osmium tetroxide for $2 \mathrm{~h}$ at $4^{\circ} \mathrm{C}$, dehydrated in graded ethanol $(30,50,70,90,100,100$ and $100 \%)$ for 15 min each at $4^{\circ} \mathrm{C}$, and embedded in Quetol 812 epoxy resin (Nisshin EM Co., Ltd.) for $48 \mathrm{~h}$ at $60^{\circ} \mathrm{C}$. Ultrathin sections (80-90 nm) were cut using an Ultracut J microtome (Reichert Jung), stained with lead nitrate and uranium acetate, and imaged using an H-7600 transmission electron microscope (Hitachi High-Technologies Corporation) at $100 \mathrm{kV}$.

Establishment of CAL27-F-XBP1- $D D B D-V e n u s$ cell lines and assessment of ER stress loading by real-time monitoring of spliced XBPl- $\triangle D B D$-Venus signal. Plasmid pCAX-Flag (F)-XBP1-lacking the DNA-binding domain ( $\triangle \mathrm{DBD}$ )-Venus (19) was a kind gift from Professor 
Masayuki Miura (Department of Genetics, Graduate School of Pharmaceutical Sciences, The University of Tokyo). The F-XBP1- $\triangle$ DBD-Venus fusion gene was subcloned into the pIRES-puro vector (modified from pIRES-EGFP-puro; Addgene, Inc.) by removing the enhanced green fluorescent protein (EGFP) sequence upstream of the puromycin resistance gene. CAL27 cells $\left(1 \times 10^{7}\right.$ cells $)$ were suspended in $100 \mu \mathrm{l}$ of Opti-MEM I (cat. no. 31985-070; Thermo Fisher Scientific, Inc.) with $10 \mu \mathrm{g}$ pIRES-F-XBP- $\triangle$ DBD-Venus-puro and transfected using the Super Electroporator NEPA 21 with a 2-mm gap cuvette (cat. no. EC-002) (both from NEPA GENE Co., Ltd.). The following conditions were used: Poring pulse (voltage, $125 \mathrm{~V}$; pulse interval, $50 \mathrm{~ms}$; pulse width, $10 \mathrm{~ms}$; pulse number, 2; attenuation rate, 10\%); and transfer pulse (voltage, $20 \mathrm{~V}$; pulse interval, $50 \mathrm{~ms}$; pulse width, $50 \mathrm{~ms}$; pulse number, 5; and attenuation rate, 40\%). After electroporation, cells were cultured in DMEM containing $10 \%$ FBS in a humidified incubator $\left(37^{\circ} \mathrm{C}, 5 \% \mathrm{CO}_{2}\right.$ and $95 \%$ air $)$ for recovery. One day after electroporation, cells were cultured in the presence of puromycin $(0.5 \mu \mathrm{g} / \mathrm{ml})$ to isolate stable transfectants. The XBP1- $\triangle \mathrm{DBD}-$ Venus fusion protein was expressed in response to $300 \mathrm{nM}$ TPG. Clone \#5 displayed the strongest fluorescence signal and was thus used for subsequent experiments.

Expression of the XBP1- $\triangle \mathrm{DBD}-$ Venus fusion protein in response to TPG was also confirmed by immunoblotting using anti-FLAG mAb (Sigma-Aldrich; Merck KGaA; cat. no. F1804; 1:1,000), anti-GFP Ab (Santa Cruz Biotechnology, Inc.; cat. no. sc-9996; 1:1,000) and anti-XBP1s (BioLegend, Inc.; cat. no. $647502 ; 1: 2,500)$ mAbs (data not shown). The established cell line was used for experiments within 10 passages of thawing. The fluorescence intensities derived from spliced XBP1-Venus were monitored using an IncuCyte ${ }^{\mathrm{TM}} \mathrm{ZOOM}$ cell imaging system (Essen BioScience) after $48 \mathrm{~h}$ of exposure to BTZ and/or RCS. At the same time, phase-contrast imaging for confluence, which calculates the density of cells in each imaging field, was performed using IncuCyte. A 10x objective lens was used in all experiments. All experiments were performed in quadruplicate using 96-well flat-bottom plates at an initial cell density of $3 \times 10^{4}$ cells $/ \mathrm{ml}$. Data analysis was performed using the IncuCyte $^{\mathrm{TM}} \mathrm{ZOOM}$ ver. $2016 \mathrm{~B}$ software (Essen BioScience).

Establishment of EGFP-LC3-mCherry-LC3AG-expressing CAL27 cells. The establishment of EGFP-LC3-mCherry-LC3A G-expressing CAL27 cells has been previously reported (18). In brief, pMRX-IP-EGFP-LC3-mCherry-LC3 $\Delta \mathrm{G}$ was electroporated into CAL27 cells using a NEPA21 Super Electroporator (NEPA GENE Co., Ltd.) as aforementioned. The transfected cells were selected with puromycin, and single clones were isolated using a cloning ring. EGFP-LC3 and mCherry-LC3 $\Delta \mathrm{G}$ expression was confirmed by fluorescence microscopy and immunoblotting using specific Abs against GFP (Santa Cruz Biotechnology, Inc.; cat. no. sc-9996; 1:1,000), mCherry (Abcam; cat. no. ab167453; 1:1,000) and LC3 (Novus Biologicals, Ltd.; cat. no. NB600-1384; 1:4,000) (18).

Assessment of reactive oxygen species (ROS) production. CAL27 cells $\left(1 \times 10^{6}\right)$ were seeded into a $60-\mathrm{mm}$ dish and pre-cultured for $24 \mathrm{~h}$. The cells were then treated with BTZ and/or RCS for 24 and $48 \mathrm{~h}$. NAC was also used in combination with these drugs as a scavenger of ROS. The cells were stained using 2',7'-dichlorodihydrofluorescein diacetate (CM-H2DCFDA; Thermo Fisher Scientific, Inc.), a chemically reduced form of cell-permeable fluorescein that is used as an indicator of ROS, according to the manufacturer's instructions. Fluorescence intensities were assessed by flow cytometry using an Attune Acoustic Focusing Cytometer, and data analysis was performed using Attune ${ }^{\circledR}$ Cytometer software version 2.1.0 (both from Thermo Fisher Scientific, Inc.).

Statistical analysis. The synergistic effect of BTZ and RCS for inhibiting cellular proliferation was statistically analyzed using Combenefit software ver. 2.021 (20). All quantitative data are expressed as the mean \pm standard deviation. One-way ANOVA followed by post-hoc tests was used to compare multiple groups; pairwise comparisons were performed using Tukey's honestly significant difference test (if all groups were to be compared) or the Games-Howell test whenever all groups were compared with a single reference group. $\mathrm{P}<0.05$ was considered to indicate a statistically significant difference.

\section{Results}

Combined treatment with BTZ and RCS synergistically induces non-apoptotic death of CAL27 and Detroit562 cells. CAL27 and Detroit562 cells were treated with BTZ (2.5, 5 and $10 \mathrm{nM})$ and/or $\operatorname{RCS}(1,2.5,5$ and $10 \mathrm{mM})$ for 24 and $48 \mathrm{~h}$. As shown in Fig. 1, both drugs inhibited the proliferation of both cell lines in a dose-dependent manner. CAL27 cells were more sensitive to RCS and BTZ than Detroit562 cells. Statistical analysis revealed prominent synergism in proliferative inhibition by combined treatment with BTZ and RCS in both cell lines. Cell morphology was examined to determine whether proliferation inhibition was mediated through a cytostatic or cytocidal effect. Although some cells treated with BTZ and RCS exhibited chromatin condensation, which is one of the characteristic features of the cells undergoing apoptosis, most of the cells were swollen with loss of plasma membrane integrity (Fig. 2A). Annexin V and PI double staining also showed that PI-positive cells were increased after combined treatment with BTZ and RCS as compared with those treated with each drug alone. An increased percentage of PI-positive cells also suggested cytocidal induction by both drugs. However, unlike staurosporine treatment (the control for apoptosis induction), only a small number of cells stained Annexin V-positive/PI-negative, which is a hallmark of cells undergoing the early phase of apoptosis (Fig. 2B). Immunoblotting with anti-caspase-3 Ab revealed the absence of caspase-3 cleavage after 24 and $48 \mathrm{~h}$ of exposure to these drugs in CAL27 cells, whereas a faint band of cleaved caspase-3 was evident in Detroit562 cells in response to BTZ and RCS (Fig. 2C). Additionally, there was no abrogation of BTZ- and RCS-induced cell death in the presence of the pan-caspase inhibitor, Z-VAD fmk (Fig. 2D). All these data indicated that apoptosis induction was not a primary contributor to the induction of cell death by BTZ and RCS. This result differed from the previous demonstration of marked synergism in the induction of apoptosis by RCS and BTZ or carfilzomib in multiple myeloma and non-Hodgkin lymphoma cells $(13,21)$. 
A

CAL27 (24 h)

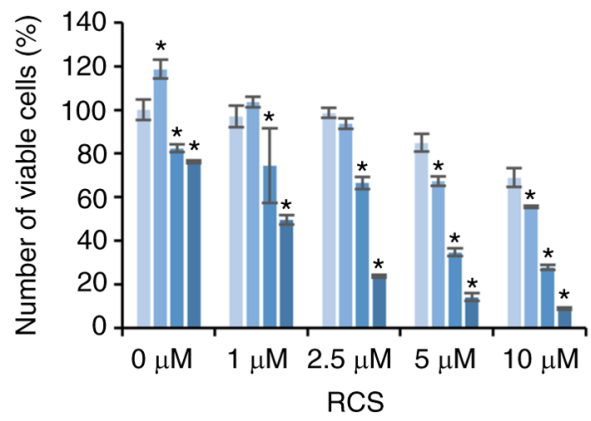

B

CAL27 (24 h)
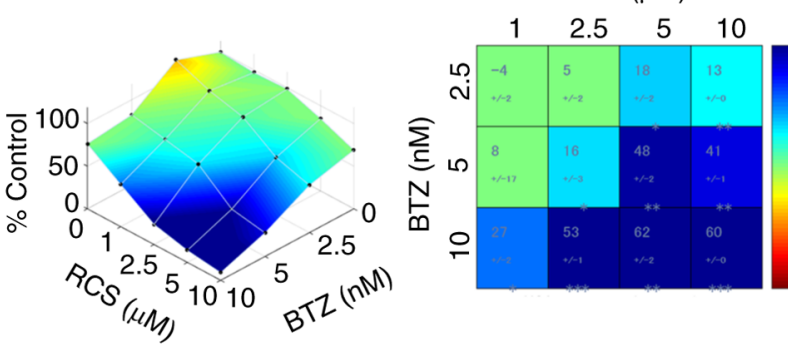

C

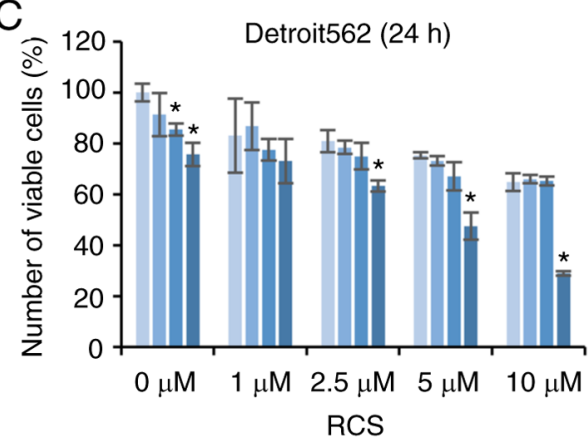

D Detroit562 $(24 \mathrm{~h})$
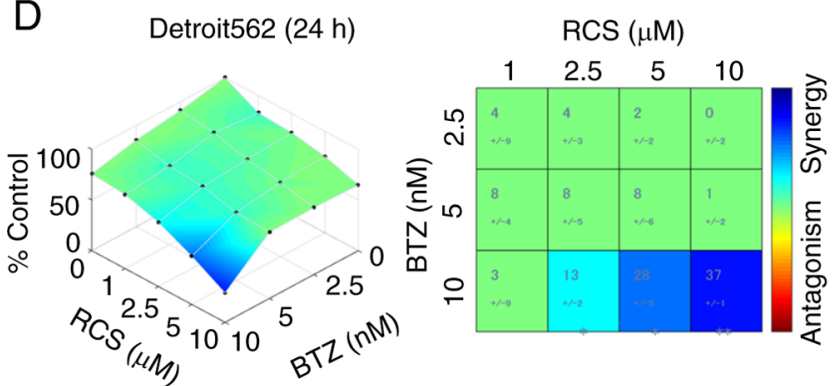

CAL27 (48 h)

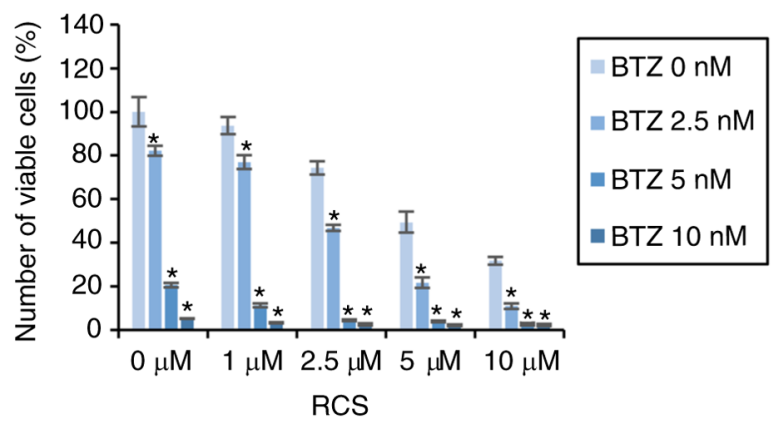

CAL27 (48 h)
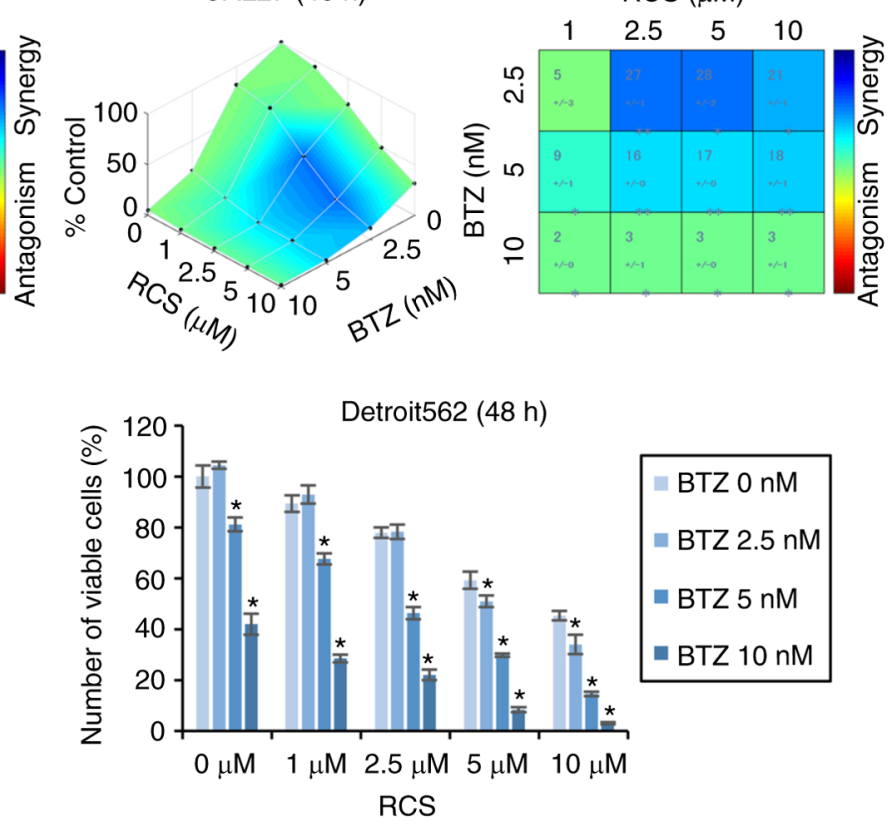

Detroit562 (48 h)

$\operatorname{RCS}(\mu \mathrm{M})$

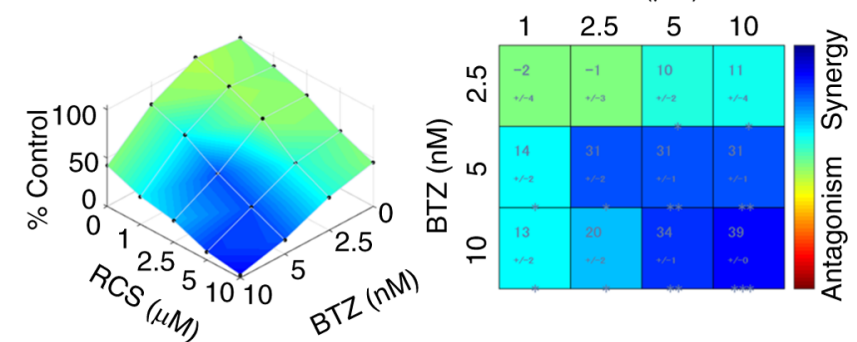

Figure 1. Synergistic cell proliferation inhibition by combined treatment with RCS and BTZ in CAL27 and Detroit562 cells. (A) CAL27 cells were treated with RCS $(1,2.5,5$ and $10 \mu \mathrm{M})$ with/without BTZ (2.5, 5 and $10 \mathrm{nM})$ for 24 and $48 \mathrm{~h}$. Viable cell number was assessed by CellTiter Blue assay. (B) Synergistic effect of CAL27 cell proliferative inhibition was statistically analyzed using Combenefit software. Left: Mapping of the synergy levels on the experimental combination dose-response surface. Right: Synergy levels in matrix format. Higher score shown in denser blue indicates a stronger synergistic effect (C and D). $\mathrm{n}=3$. Data are presented as the mean $\pm \mathrm{SD}$. " $\mathrm{P}<0.05 \mathrm{vs}$. BTZ $0 \mathrm{nM}$. Detroit562 cells were treated with RCS and BTZ, and the synergistic effect of cell growth inhibition was statistically analyzed as aforementioned. RCS, ricolinostat; BTZ, bortezomib.

Induction of autophagy following treatment with BTZ and RCS. Our previous studies reported that BTZ induced autophagy in myeloma and breast cancer cells $(11,15)$. Tubacin, an HDCA6 inhibitor, reportedly induces the suppression of autophagy and blocks the fusion of autophagosomes, which may overcome drug resistance in glioma cells (22). By contrast, the pan-HDAC inhibitor SAHA reportedly induces autophagy in glioblastoma cells (23). Thus, the induction of autophagy in response to HDCA6 inhibitors appears not to be consistent. To determine whether RCS induced or inhibited autophagy, and to clarify whether combined treatment with BTZ and RCS induced autophagic cell death, an autophagy flux assay was performed using CAL27 cells stably expressing the GFP-LC3-mCherry-LC3 $\Delta \mathrm{G}$ probe $(18,24)$. The induction of autophagy displayed a downward curve as evidenced by the reduction in the $\mathrm{GFP} / \mathrm{mCherry}$ ratio, as was the case of cells treated with rapamycin. By contrast, blocking autophagy flux produced an upward curve due to the increase 
A
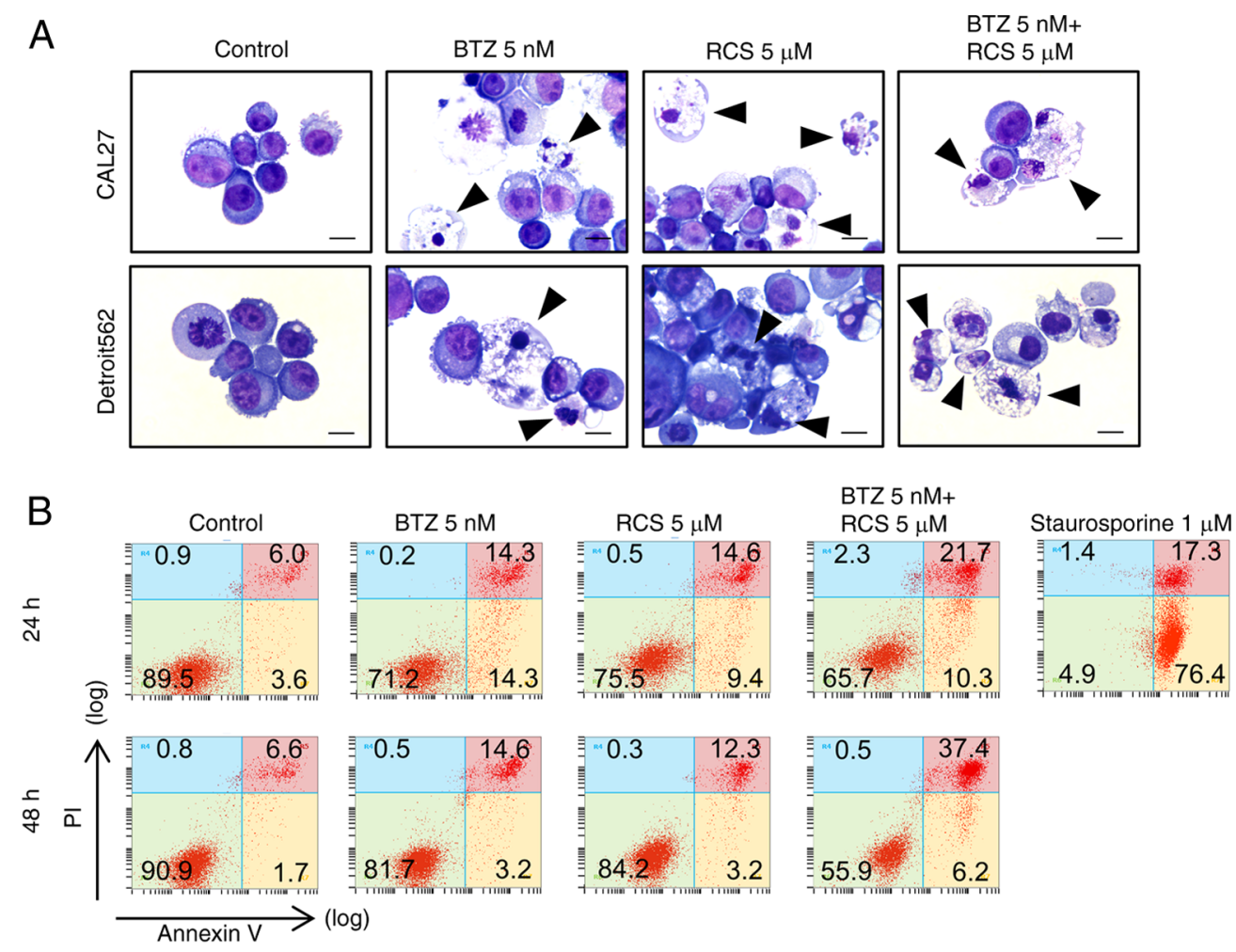

\section{Detroit562}

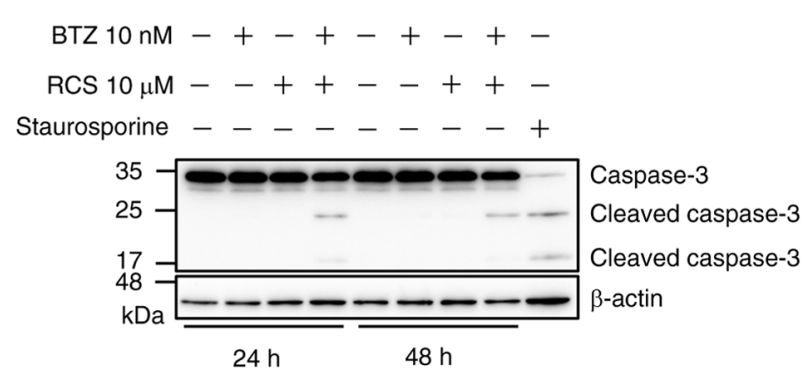

D

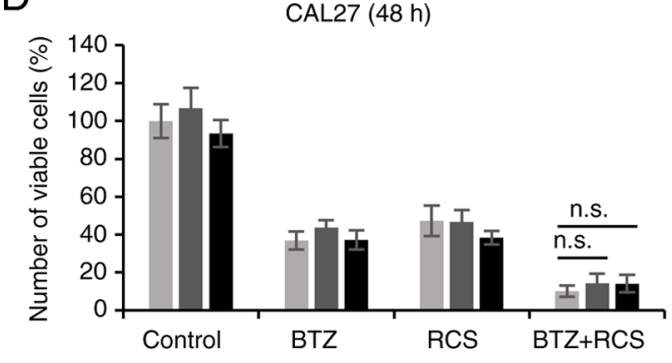

CAL27 (48 h)

BTZ $5 \mathrm{nM}-+-+-+-+-$

$\operatorname{RCS} 5 \mu \mathrm{M}--++--++$

Staurosporine - - - - - - +

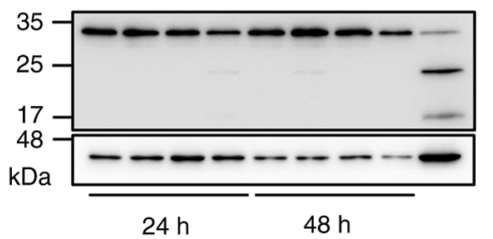

Figure 2. Induction of non-apoptotic cell death after treatment of CAL27 and Detroit562 cells with BTZ plus RCS. (A) CAL27 cells were treated with either BTZ $(5 \mathrm{nM})$, RCS $(5 \mu \mathrm{M})$, or BTZ plus RCS for $24 \mathrm{~h}$, and stained with May-Grünwald-Giemsa. Magnification, x100. Scale bar, $20 \mu \mathrm{m}$. Arrowheads indicate nuclear chromatin condensation. (B) After treatment with either BTZ, RCS, or BTZ plus RCS for 24 and 48 h, CAL27 cells were examined by flow cytometry following Annexin V and PI double staining. Number of each area indicates the percentage of cells. Treatment with $1 \mu \mathrm{M}$ staurosporine at for $4 \mathrm{~h}$ was a positive control for apoptosis induction. (C) CAL27 and Detroit562 cells were treated with/without BTZ in the presence or absence of RCS for 24 and $48 \mathrm{~h}$. Immunoblotting was performed using anti-caspase-3 mAb. (D) After treatment with BTZ, RCS, and BTZ plus RCS in the presence of Z-VAD fmk $(25$ or $50 \mu \mathrm{M})$ for $48 \mathrm{~h}$, viable cell numbers were determined by the CellTiter Blue assay. RCS, ricolinostat; BTZ, bortezomib; n.s., not significant.

in the GFP/mCherry ratio, as found with cells treated with bafilomycin A1 (Figs. 3A and S1). Both BTZ and RCS induced a downward trend due to a reduction in the GFP/mCherry ratio. These findings indicated induction of autophagy in response to both drugs (18). The combination of BTZ and RCS still induced autophagy, as demonstrated by a downward curve, but did not markedly enhance autophagic induction compared with the results produced by each drug alone. TEM revealed that BTZ treatment increased the number of autophagosomes in perinuclear region (Fig. S2). Furthermore, after $24 \mathrm{~h}$ of treatment with RCS, there was an apparent increase in the number of autophagosomes and lysosomes in the cytoplasm. 
Combined treatment with BTZ and RCS resulted in prominent autolysosome formation along with engulfment of lysosomes, which appeared to be due to pronounced lysophagy (Figs. 3B and S2) (25). These data suggest that both BTZ and RCS induce autophagy in CAL27 cells.

In the presence of the 3-MA autophagy inhibitor, cell viability remained unchanged compared with BTZ, RCS, or BTZ + RCS treatment alone. The findings suggested that autophagic cell death and type-II programmed cell death (PCD) did not contribute to the pronounced lethality induced by BTZ plus RCS (Fig. 3C) (26,27).

Necroptosis-like cell death following combined treatment with BTZ and RCS. The aforementioned data indicated the induction of non-apoptotic and non-autophagic cell death following treatment with BTZ and RCS. Notably, the presence of the necrostatin RIPK-1 inhibitor significantly suppressed the enhanced cytotoxicity of BTZ plus RCS in both cell lines, strongly suggesting the induction of necroptosis (Fig. 4A) (28). However, phosphorylated-RIPK1 and phosphorylated-MLKL could not be detected after treatment with BTZ plus RCS (Fig. 4B) (28-30). Therefore, RIPK1-dependent necroptosis could not conclusively identified.

RCS inhibits BTZ-induced aggresome formation in CAL27 cells and induces ER stress loading. Intracellular misfolded proteins are transported on the microtubule by the dynein motor protein, to the microtubule organization center of the perinuclear region; this results in aggresome formation in a process that is dependent on the deacetylation of $\alpha$-tubulin $(6,31)$. In the current study, immunofluorescence and immunoblotting indicated that cytoplasmic tubulin was acetylated in response to RCS treatment (Figs. 5A and S3). Aggresome formation is accompanied by the redistribution of an intermediate filament protein, vimentin, to form a cage surrounding a pericentriolar core of aggregated and ubiquitinated protein $(32,33)$. Our previous study reported that BTZ-induced aggresome formation is detected as a vimentin-positive perinuclear inclusion body in MDA-MB231 metastatic breast cancer cells (34). However, because CAL27 cells were vimentin-negative, and the intermediate filament that is required for aggresome formation in CAL27 cells is yet to be identified, ubiquitinated proteins (that are concentrated in the aggresome) were detected in the present study. Treatment with $5 \mathrm{nM}$ BTZ for $24 \mathrm{~h}$ resulted in the formation of several Ub-positive puncta, while $10 \mathrm{nM}$ BTZ resulted in an enlarged Ub-positive aggregate body in the perinuclear region, which may indicate aggresome formation (Fig. 5B). Treatment with $10 \mu \mathrm{M}$ RCS did not have any effect on the ubiquitin staining pattern, compared with untreated control cells. However, large BTZ-induced Ub-aggregates in perinuclear regions were markedly reduced in the presence of RCS. Furthermore, BTZ treatment increased the amount of cytoplasmic ubiquitinated proteins in the detergent-soluble fraction, as well as in the detergent-insoluble fractions in CAL27 cells (Fig. 5C). In the presence of RCS, these ubiquitinated proteins were further increased, along with a reduction in the insoluble fraction. These data suggest that RCS inhibited BTZ-induced aggresome formation, leading to an increase in cytoplasmic ubiquitinated proteins following proteasome inhibition.
Our previous studies reported that aggresome formation in response to proteasome inhibition plays a role in attenuating ER stress loading $(16,34)$. Thus, in the present study, ER stress loading was assessed following treatment with BTZ and RCS, using immunoblotting and a real-time ER stress monitoring system (16). Immunoblotting revealed that BTZ or RCS treatment did not alter the expression of GRP78, while expression was increased by combined treatment with BTZ and RCS (Fig. 6A). Additionally, the real-time quantitative ER stress loading analysis indicated enhanced ER stress loading by concomitant exposure to BTZ and RCS (Fig. 6B). However, the pronounced induction of CHOP (GADD153), which is an ER stress-related pro-apoptotic transcription factor, could not be detected (Fig. 6A) (35). These data suggest that the combination of BTZ and RCS enhanced ER stress loading, which contribute to increased cell death. However, this ER stress loading did not indicate an upregulation the pro-apoptotic transcription factor $\mathrm{CHOP}$, as in the case of myeloma and breast cancer cells $(11,15,16)$. This result appeared to be consistent with the induction of non-apoptotic cell death in response to BTZ and RCS (Fig. 2).

Enhanced ROS production by combined treatment with BTZ and RCS. A vital mediator of ER stress-mediated cell death is ROS generation by the ER and mitochondria. Therefore, intracellular ROS levels were evaluated. Using an H2DCFDA probe to monitor ROS production, pronounced ROS production was observed following BTZ plus RCS treatment for $48 \mathrm{~h}$, compared with that produced by each agent alone (Fig. 7A). Furthermore, the NAC ROS scavenger inhibited the enhanced cytotoxic effect of combined treatment with RCS and BTZ (Fig. 7A and B), which strongly supports the involvement of ROS in cell death by these drug combinations. This demonstrates that ER stress loading and ROS production are involved in necroptosis-like cell death induced by combined treatment with BTZ and RCS.

\section{Discussion}

The present study demonstrated that combined treatment with a proteasome and HDCA6 inhibitor synergistically reduced the viability of CAL27 and Detroit562 head and neck squamous cancer cells. To the best of our knowledge, the study is the first to report the combined effects of BTZ and RCS on squamous cancer cells, if similar effects were confirmed in in vivo experiments, this enhanced cytotoxicity may be promising in a clinical setting. The results are partially consistent with previous reports in multiple myeloma and non-Hodgkin lymphoma cells $(13,21)$. Although myeloma cells exhibited typical apoptosis (type-I PCD) when treated with RCS and carfilzomib at almost equivalent drug concentrations as in this study, CAL27 and Detroit562 cells exhibited a non-apoptotic phenotype (13) There were no typical morphological features of apoptosis, such as nuclear fragments and apoptotic bodies, caspase-3 activation, abrogation of the induction of cell death induction in the presence of a pan-caspase inhibitor, and Annexin V-positive/PI-negative cells by flow cytometry. Since CAL27 and Detroit562 cells undergo apoptosis in response to various conditions, including staurosporine treatment, molecular evidence of apoptosis can be detected in these cell 
A

CAL27_GFP-LC3-mCherry-LC3 $\Delta G$

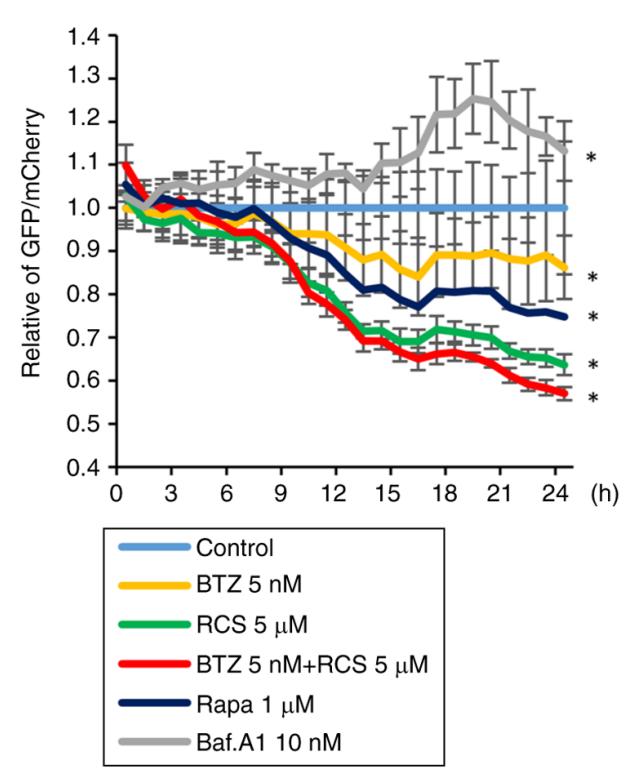

C

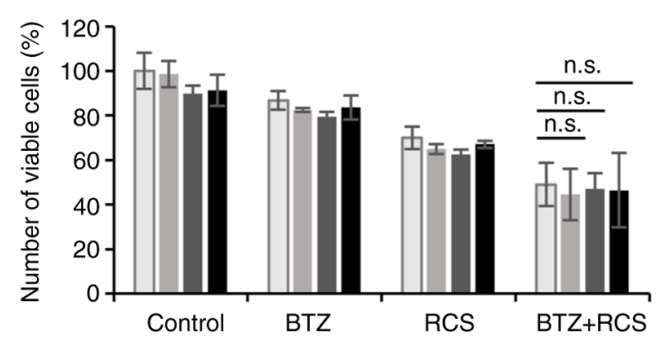

Detroit562 (24 h)

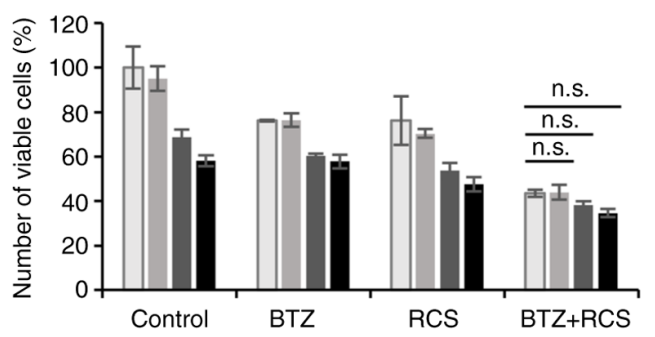

B

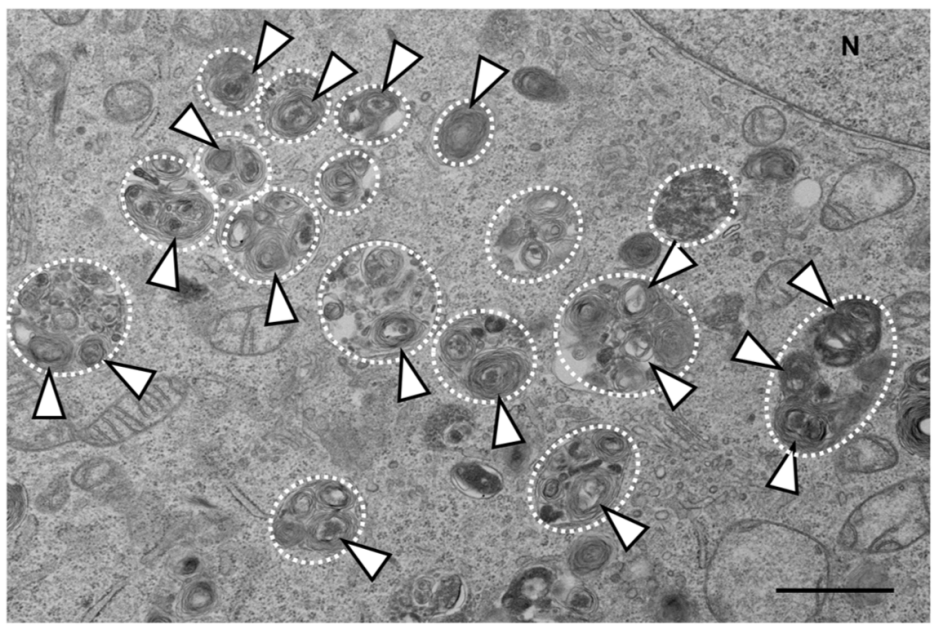

CAL27 (48 h)

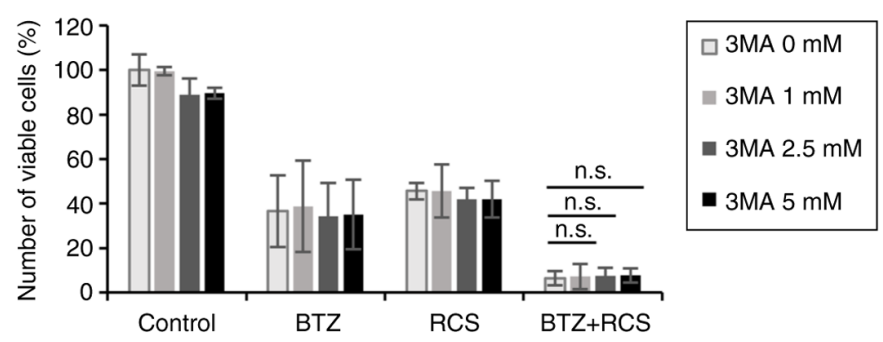

Detroit562 (48 h)

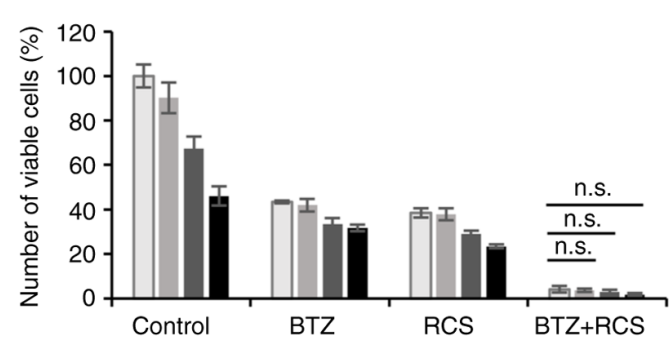

$\square 3 \mathrm{MA} 0 \mathrm{mM}$

- 3MA $1 \mathrm{mM}$

- 3MA $2.5 \mathrm{mM}$

- 3MA 5 mM

Figure 3. Assessment of autophagy induction in CAL27 cells by treatment with BTZ and RSC.(A) Autophagy flux assay using CAL27-GFP-LC3-mcherry-LC3 $\triangle$ G was performed as described in Materials and methods. The GFP/mCherry ratios were normalized to those of the cells cultured in the control medium defined as 1.0 at each time point and were and plotted as the mean $\pm \mathrm{SD}$. Treatment with $1 \mu \mathrm{M}$ Rapa was used as a control for autophagy induction, and $10 \mathrm{nM}$ Baf.A1 as a control for autophagy inhibitor. (B) Transmission electron microscopy of CAL27 cells after treatment with BTZ and RCS for $24 \mathrm{~h}$. Scale bar, $1 \mathrm{~mm}$. Dashed circle indicates autolysosome. Arrowheads indicate the engulfed lysosome. (C) After treatment with BTZ, RCS, and BTZ plus RCS in the presence of 3MA $(1,2.5$, and $5 \mathrm{mM})$, viable cell numbers were determined by the CellTiter Blue assay. " $\mathrm{P}<0.05$ vs. control cells. RCS, ricolinostat; BTZ, bortezomib; n.s., not significant; 3MA, 3-methyladenine; Rapa, rapamycin; Baf.A1, bafilomycin A1.

lines. To clarify the molecular mechanism underlying the synergistic cytotoxicity of the BTZ and RCS drug combination, an attempt to identify the cell death phenotype involved in this pronounced cytotoxicity was made.

Combined treatment with BTZ and RCS induced autophagy. However, the involvement of autophagic cell death (type-II PCD) in CAL27 and Detroit562 cells was excluded $(26,28)$. Notably, abrogation of prominent cytotoxicity was observed in the presence of the RIPK1 inhibitor necrostatin-1. This finding strongly indicated the induction of necroptosis (type-III PCD), which was supported by the observed morphological features, as well as an increased number of annexin V-positive/PI-positive cells. The accumulation of autophagosomes plays a role in the formation of necrosomes composed of p62, RIPK1, RIPK 3 and MLKL (for necroptosis induction), rather than apoptosis (36). RIPK3-mediated MLKL phosphorylation within the necrosome results in the formation of an MLKL octamer, and recruitment of the octamer to the plasma membrane creates a pore on the membrane for necroptosis (30). However, RIPK1 or MLKL phosphorylation were not detected in the present study, and low expression of RIPK3 in CAL27 cells has previously been described (37). Additionally, although BTZ 
A

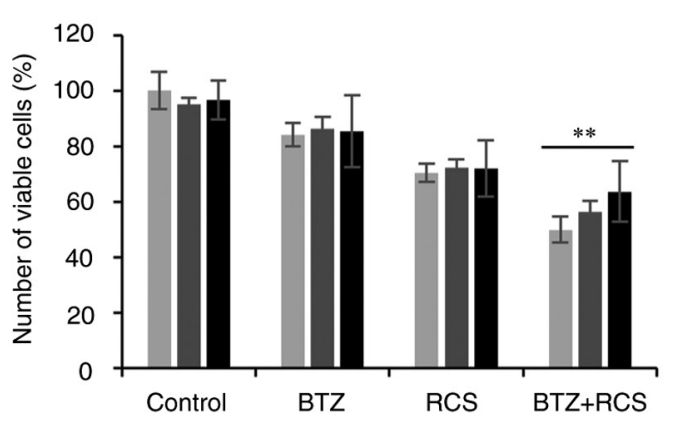

Detroit562 (24 h)

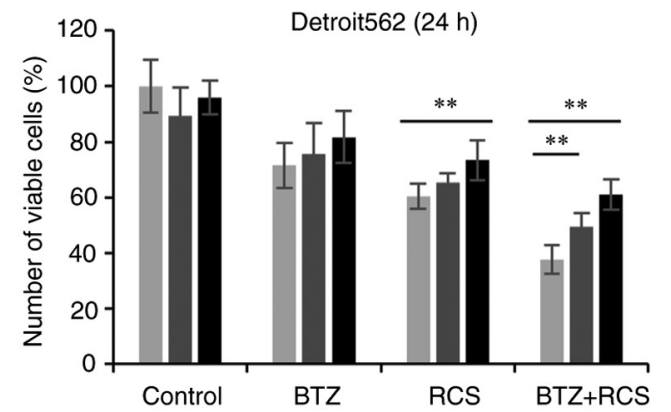

B
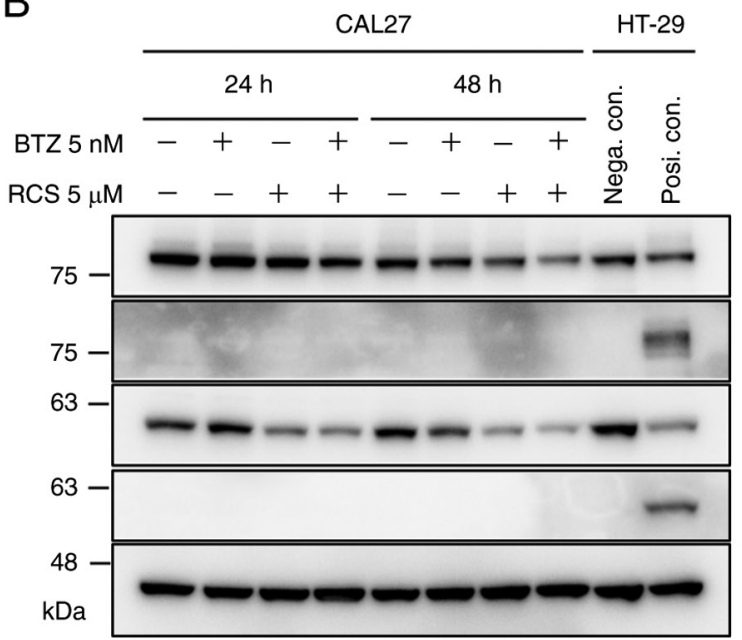

CAL27 (48 h)
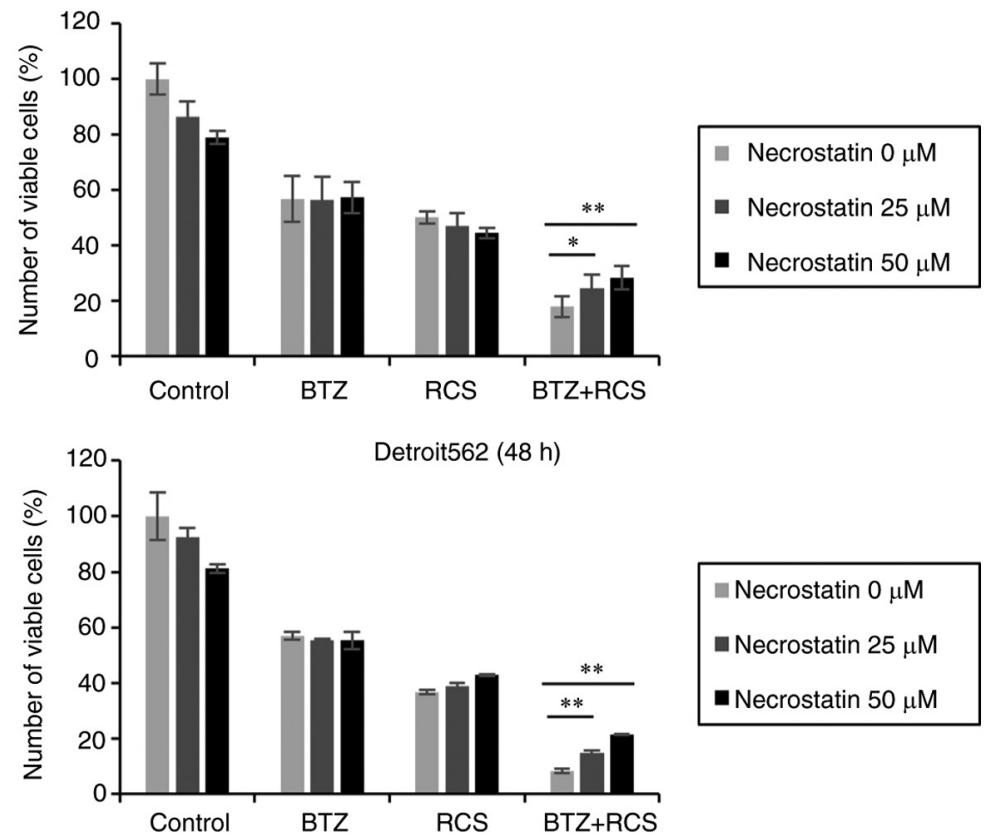

Figure 4. Necrostatin-1 and NAC suppress cytotoxicity enhanced by BTZ plus RCS in CAL27 and Detroit562 cells. (A) CAL27 and Detroit562 cells were untreated or treated with BTZ ( $5 \mathrm{nM}$ for CAL27 cells, $10 \mathrm{nM}$ for Detroit562 cells) and/or RCS (5 $\mu \mathrm{M}$ for CAL27 cells, $10 \mu \mathrm{M}$ for Detroit562 cells) in the presence or absence of necrostatin-1 ( 25 and $50 \mu \mathrm{M})$ for 24 and $48 \mathrm{~h}$. Viable cell number was determined by the CellTiter Blue assay. ${ }^{*} \mathrm{P}<0.05$ and ${ }^{* * *} \mathrm{P}<0.01$ vs. cells not treated with necrostatin-1. (B) CAL27 and Detroit562 cells were untreated or treated with BTZ in the presence or absence of RCS for 24 and $48 \mathrm{~h}$ followed by immunoblotting. HT-29 cells treated with Z-VAD-fmk, cycloheximide and TNF- $\alpha$ were a positive control for induction of necroptosis. Immunoblotting with anti- $\beta$-actin mAb was performed as a loading control. NAC, N-acetyl-L-cysteine; RCS, ricolinostat; BTZ, bortezomib; RIPK1, serine/threonine-protein kinase 1; MLKL, mixed lineage kinase domain-like protein.

plus RCS increased the content of autophagosomes, which can be a scaffold for necrosomes, inhibition of autophagosome formation by 3-MA did not suppress cytotoxicity. Therefore, the canonical molecular machinery for necroptosis induction, cell death induction using autophagosomes as a scaffold, does not appear to function in this case. Taken together, the pronounced cytotoxicity induced by combined BTZ and RCS treatment appeared to be mediated through necroptosis-like cell death without RIPK1 and MLKL activation. The precise molecular mechanism remains to be elucidated.

The most significant points in the present study are the contribution of ROS and ER stress loading to cell death induction, and the mutual association between ROS and
ER stress. ER stress was increased by RCS, along with the suppression of BTZ-induced aggresome formation, and appeared to be the same as observed in myeloma and breast cancer cells $(13,16)$. However, upregulation of the ER stress-related pro-apoptotic transcription factor $\mathrm{CHOP}$ could not be detected in response to BZ and RCS. In a breast cancer cell line stably expressing pEZ-F-XBP1-Venus, combined treatment with BTZ and SAHA resulted in a marked increase in ER stress loading, leading to the upregulation of $\mathrm{CHOP}$, which in turn resulted in enhanced induction of apoptosis $(11,16)$. In myeloma cells, concomitant treatment with BTZ and HDAC6-knockdown resulted in enhanced CHOP expression following the upregulation of Bim and DR5 to induce 
A
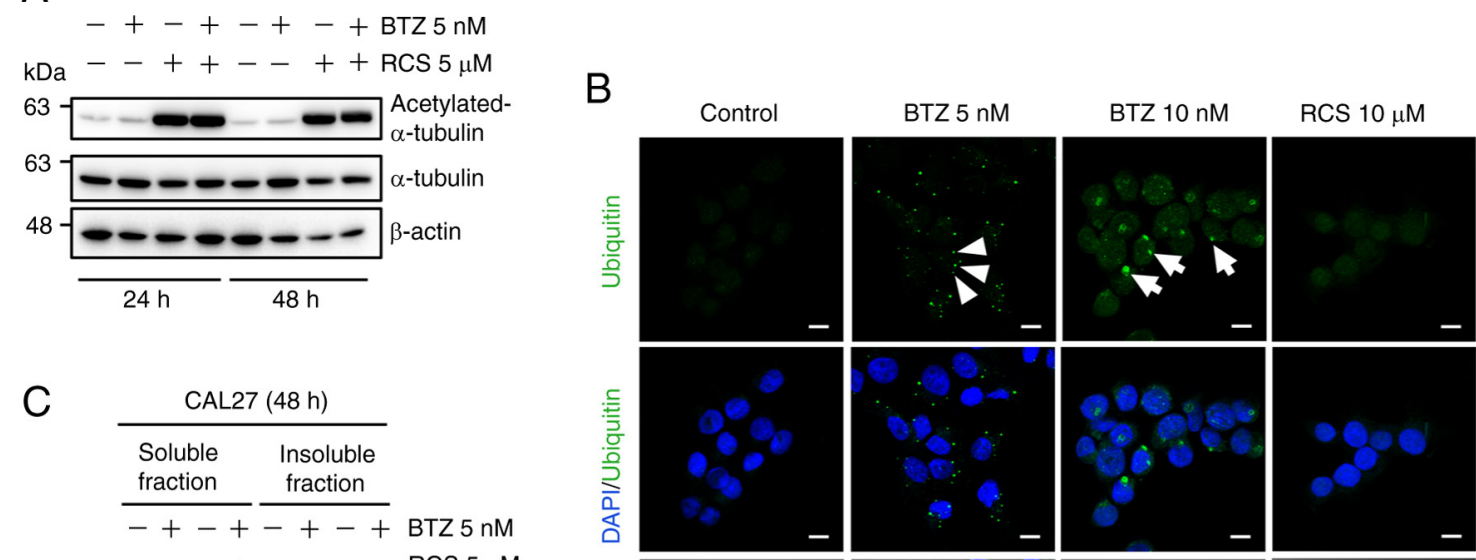

BTZ $10 \mathrm{nM}+$
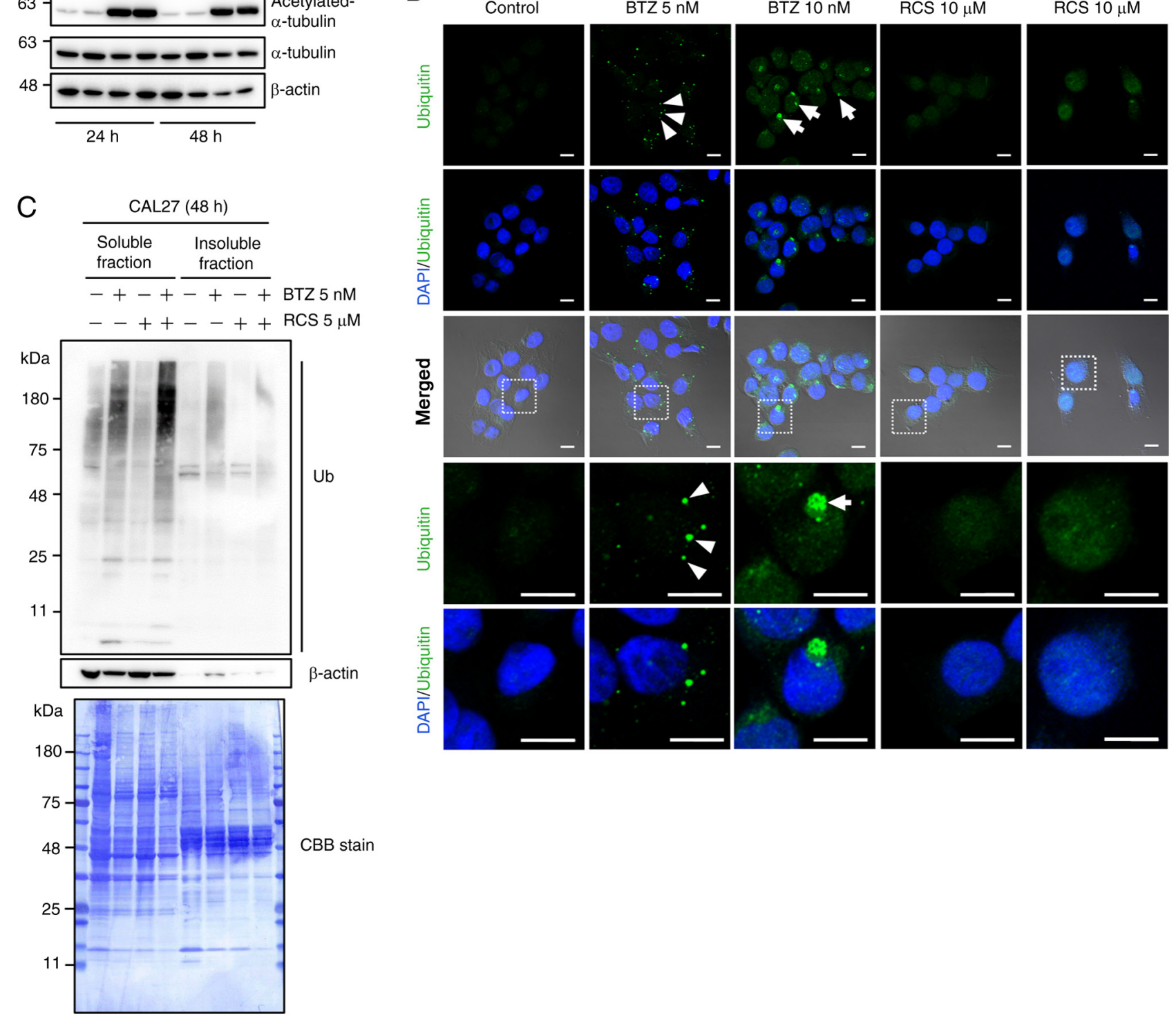

Figure 5. RCS inhibits BTZ-induced cytoplasmic ubiquitin condensation in CAL27 cells. (A) CAL27 cells were treated with BTZ (5 nM) and/or RCS (5 $\mu$ M) for 24 and $48 \mathrm{~h}$. Immunostaining using anti-acetylated tubulin and anti-tubulin mAbs was performed. Immunoblotting with anti- $\beta$-actin mAb was performed as a loading control. (B) CAL27 cells were treated with BTZ (5 and $10 \mathrm{nM})$ and/or RCS $(10 \mu \mathrm{M})$ for $24 \mathrm{~h}$. Fluorescence immunostaining was performed with anti-ubiquitin (Ub) $\mathrm{mAb}$. Arrowheads indicate the speckled pattern of Ub-proteins. Arrows indicate the aggregate of Ub-proteins. DAPI staining indicates the position of the nucleus (blue). Scale bar, $10 \mu \mathrm{m}$. (C) Immunoblotting with anti-Ub mAb in CAL27 cells after treatment with BTZ (5 nM) and/or RCS (5 $\mu \mathrm{M})$ for $48 \mathrm{~h}$ (top). Immunoblotting with anti- $\beta$-actin $\mathrm{mAb}$ was performed as an internal control. Same membrane was subsequently stained with $\mathrm{CBB}$ to compare protein loading amounts (bottom). RCS, ricolinostat; BTZ, bortezomib; mAb, monoclonal antibody; Ub, ubiquitin; CBB, Coomassie Brilliant Blue.

apoptosis (38). These results implicate ER stress loading as the direct underlying mechanism for the pronounced cytotoxicity in breast cancer and myeloma cells. However, unlike these cases, non-apoptotic cell death without CHOP upregulation was observed in the present study, which appears to be unique to head and neck squamous cell carcinoma. Additionally, this pronounced cytotoxicity was abrogated in the presence of the ROS scavenger NAC, indicating the contribution of ROS to the synergistic cytotoxicity.

Regarding the association between ROS and ER stress, ROS are generated in multiple organelles, including the mitochondria and ER, as a byproduct of mitochondrial respiration, oxidative protein folding and detoxification. It is estimated that $\sim 25 \%$ of ROS are generated in the ER during oxidative protein folding, the formation and isomerization of disulfide bonds (39). Altered redox homeostasis in the ER causes ER stress, which in turn induces ROS production (40). Moreover, the ER is a major site of calcium storage (41). Calcium from ER cisternae primarily flows through calcium release channels, such as inositol 1,4,5-trisphosphate and ryanodine receptors, which accumulate in mitochondrial-associated membranes. The released calcium ions enter the mitochondria through voltage-dependent anion channels or calcium uniporters. High levels of calcium stimulate respiratory chain 
A
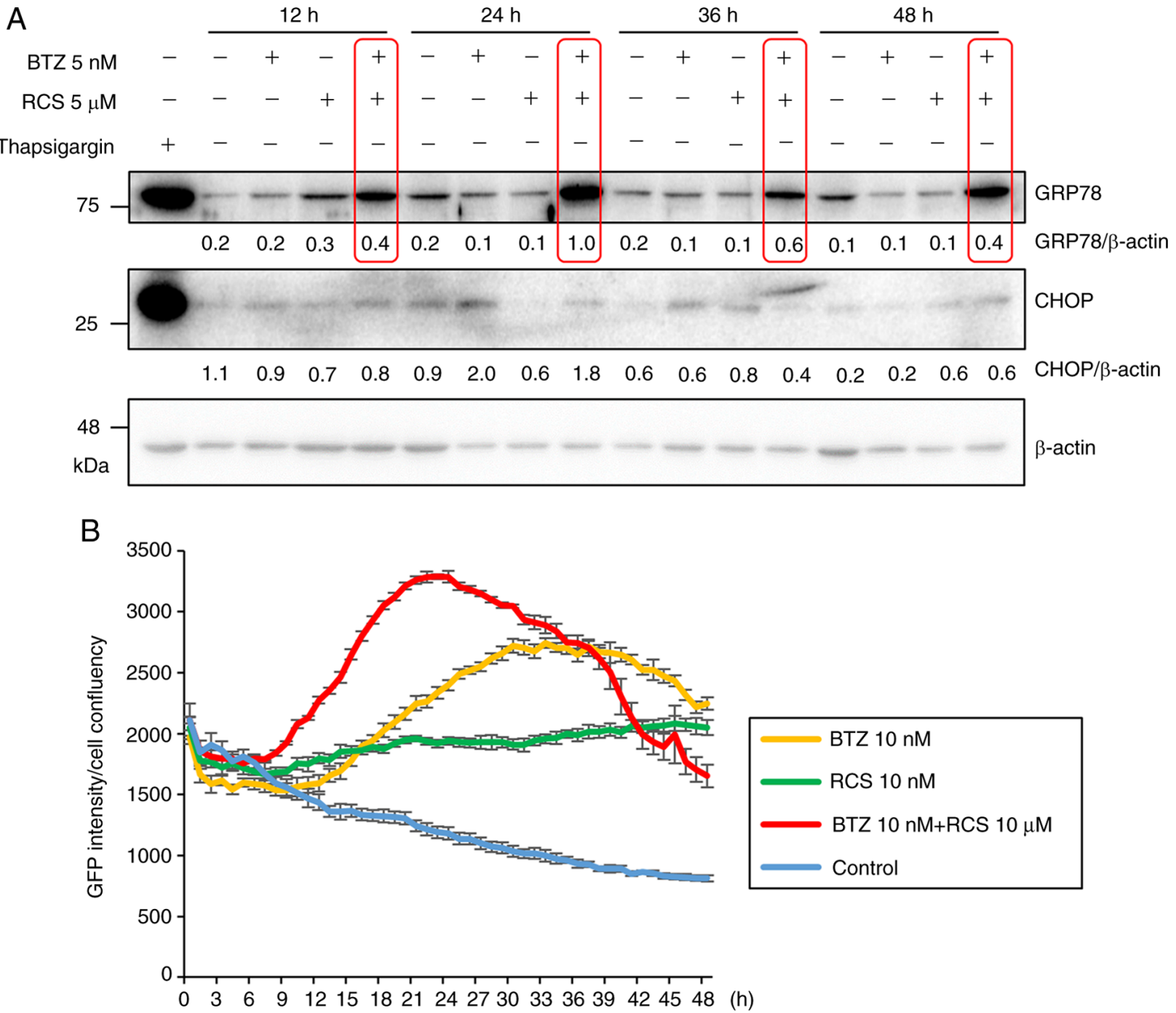

Figure 6. Enhanced ER stress loading by combined treatment with BTZ and RCS in CAL27 cells. (A) Immunoblotting with GRP78 mAb and CHOP mAb of cell lysates of CAL27 cells obtained following culture under the complete culture condition with/without BTZ ( $5 \mathrm{nM})$ in the presence or absence of RCS ( $5 \mu \mathrm{M})$ for $12,24,36$ and $48 \mathrm{~h}$. Treatment with thapsigargin at $300 \mathrm{nM}$ for $24 \mathrm{~h}$ was performed as a positive control for ER stress loading. Immunoblotting with anti- $\beta$-actin $\mathrm{mAb}$ was used as an internal control. Numbers indicate the ratios of the GRP78 to $\beta$-actin and the CHOP to $\beta$-actin in each lane. (B) CAL27-XBP1-Venus cells were treated with BTZ $(10 \mathrm{nM})$ and with/without RCS $(10 \mu \mathrm{M})$. ER stress loading was quantitatively monitored during a 48 -h exposure to these drugs. RCS, ricolinostat; BTZ, bortezomib; mAb, monoclonal antibody; ER, endoplasmic reticulum.

activity, leading to higher ROS production in the mitochondria. These ROS can further target ER-based calcium channels, resulting in increased release of calcium and a further increase in ROS levels (41). Thus, under ER stress loading in response to BTZ and RCS, the positive-feedback loop may be involved in ROS generation in both the ER and the mitochondria. A dopamine receptor antagonist, thioridazine, reportedly induced Bax-Bak-dependent and -independent apoptosis in cancer cells by enhancing ROS production followed by ER stress (42).

The findings of the present study suggest that dual targeting of the proteasome and HDAC6 induces potent cell death in head and neck squamous cell carcinoma cells. This effect appears to be mediated through the overgeneration of ROS along with ER stress loading. Therefore, in addition to patients with multiple myeloma, the BTZ plus RCS combination may be promising as a therapeutic option for patients with head and neck tumors. However, either necrosis or necroptosis-like cell death releases cellular contents, which may evoke inflammatory reactions, as well as adverse events (43). Further studies are required regarding the effects on the tumor microenvironment, and in vivo effects, for clinical application.

\section{Acknowledgements}

The authors would like to thank Ms. Ayako Hirota (Department of Biochemistry, Tokyo Medical University) for her assistance in preparing the manuscript.

\section{Funding}

The present study was supported by funds provided by the Strategic Research Foundation at Private University (grant no. S1411011 and 2014-2018) from the Ministry of Education, Culture, Sports, Science, and Technology (MEXT) of Japan awarded (grant no. 17K15032). 
A

CAL27 (24 h)

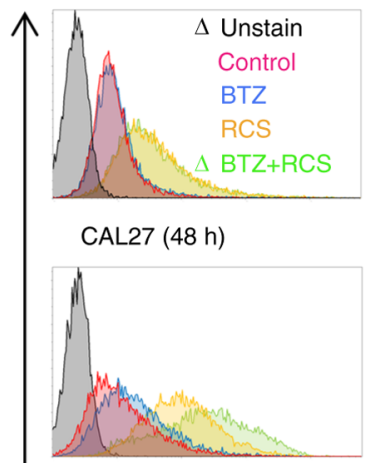

Detroit562 (24 h)

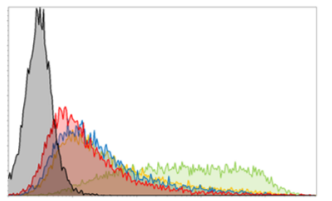

Detroit562 (48 h)

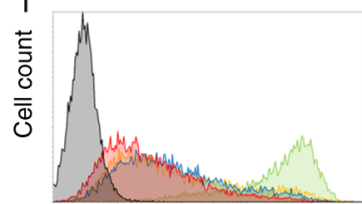

BL1-H
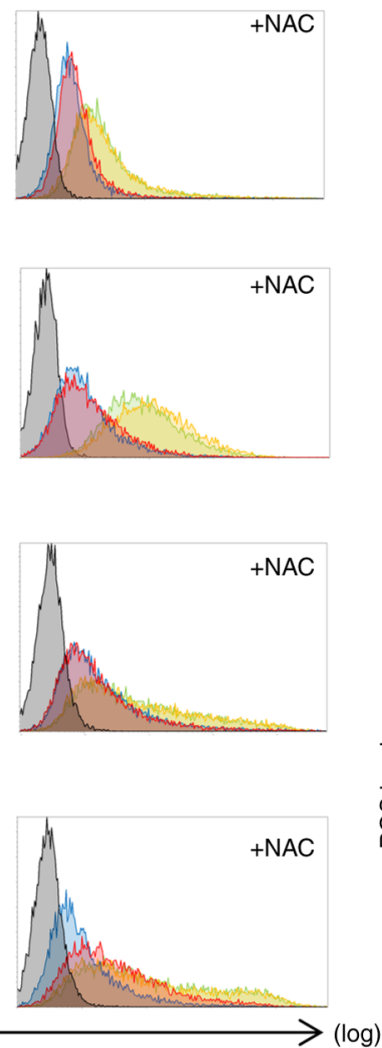

CAL27

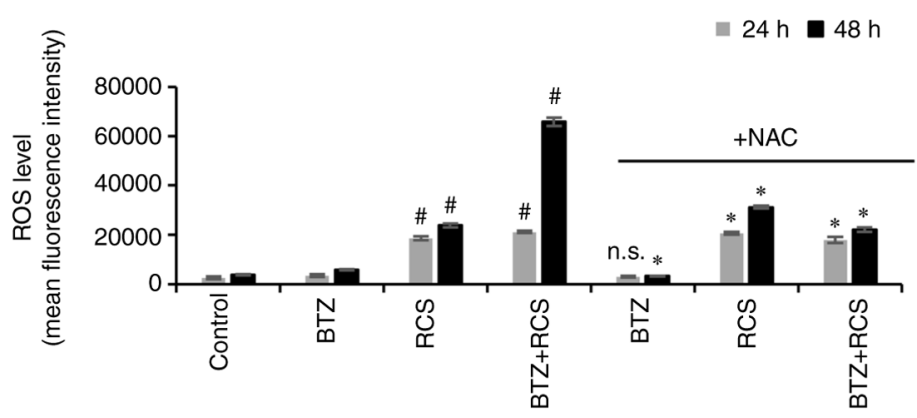

Detroit562

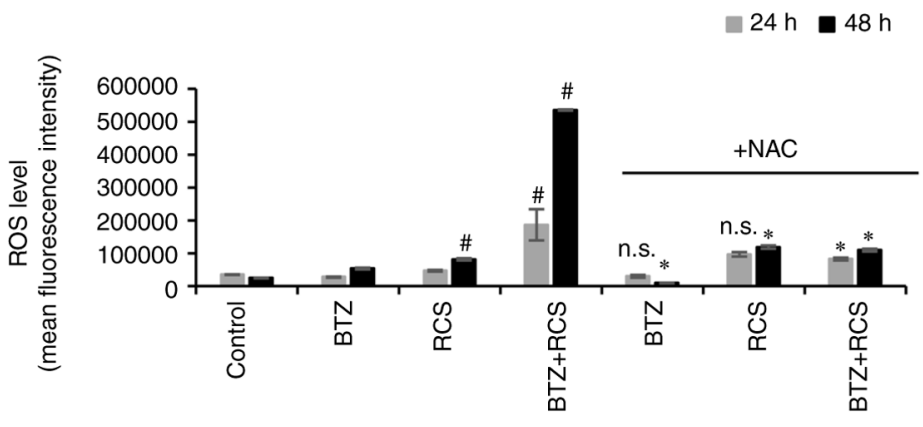

$\mathrm{B}$

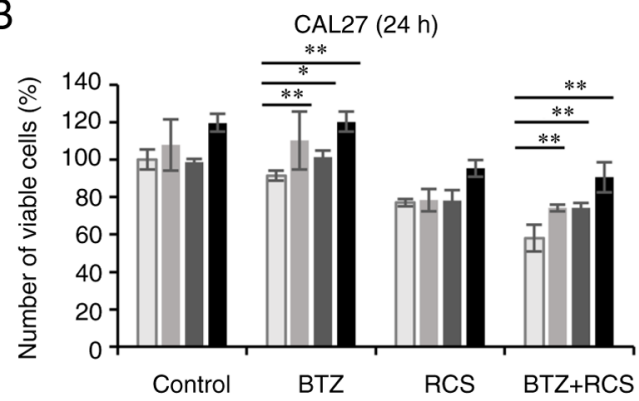

Detroit562 (24 h)

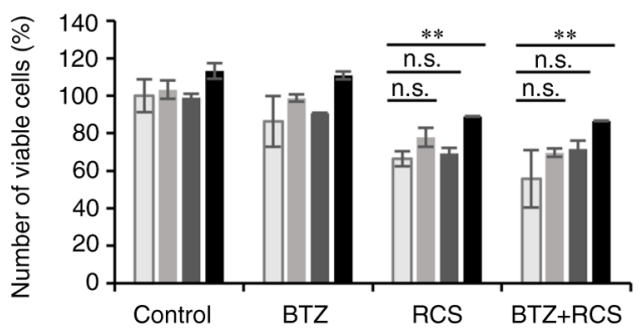

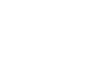
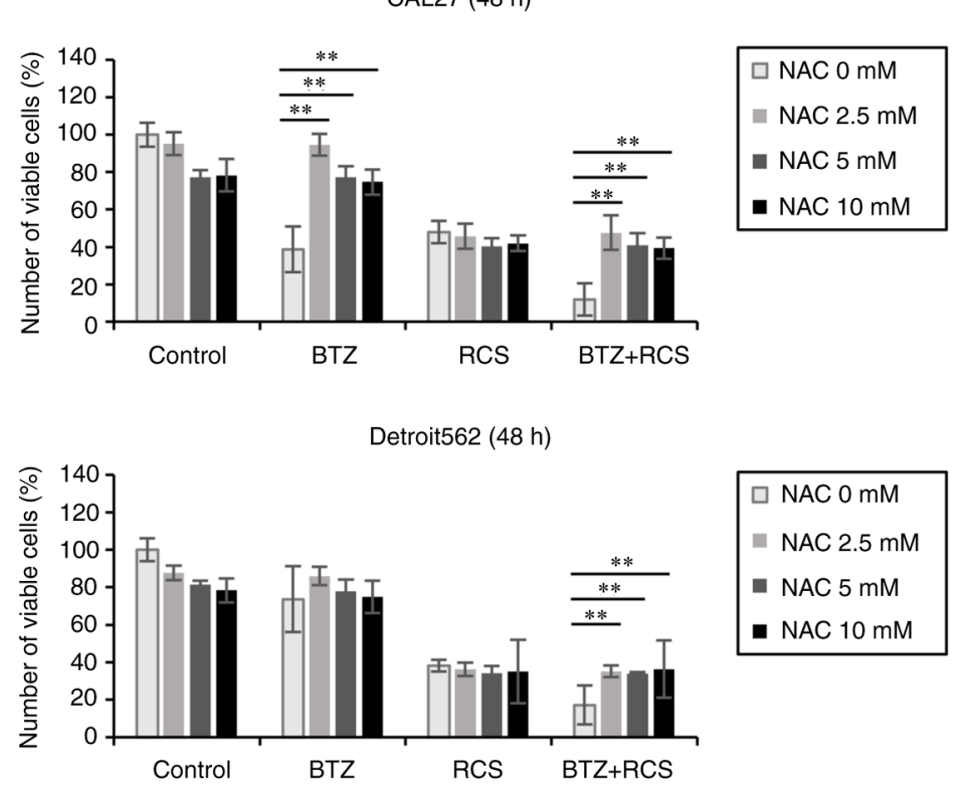

Figure 7. Enhanced ROS production after exposure to BTZ plus RCS in CAL27 and Detroit562 cells. CAL27 and Detroit562 cells were treated with BTZ (5 nM for CAL27 cells, $10 \mathrm{nM}$ for Detroit562 cells) and/or RCS ( $5 \mu \mathrm{M}$ for CAL27 cells, $10 \mu \mathrm{M}$ for Detroit562 cells) in the presence or absence of NAC (2.5, 5 and $10 \mathrm{mM}$ ). (A) ROS production was evaluated by H2DCFDA staining. Fluorescence intensities were assessed by flow cytometry. The mean \pm SD of the CM-H2DCFDA fluorescent signals after 24 - and 48 -h treatment are displayed. ${ }^{*} \mathrm{P}<0.05$ and ${ }^{* * *} \mathrm{P}<0.01$ vs. cells not treated with NAC. ${ }^{*} \mathrm{P}<0.05$ vs. control without NAC. (B) Viable cell number was assessed using CellTiter Blue. ${ }^{*} \mathrm{P}<0.05$ and ${ }^{* *} \mathrm{P}<0.01$ vs. cells not treated with NAC. RCS, ricolinostat; BTZ, bortezomib; ROS, reactive oxygen species; NAC, N-acetyl-L-cysteine.

\section{Availability of data and materials}

The datasets used and/or analyzed during the current study are available from the corresponding author on reasonable request.

\section{Authors' contributions}

KeisM and NT designed the experiments. KH performed most of the experiments and analyzed the data. NT, HK, SM and KeitM assisted $\mathrm{KH}$ in the acquisition and analysis of data. $\mathrm{KH}$ 
and NT confirmed the authenticity of all the raw data. KeisM, $\mathrm{NT}, \mathrm{MH}$ and KT were involved in the conception and mentoring, as well as in critical interpretation and evaluation of the data. KeisM, NT, MH, and KT were involved in writing, reviewing and editing the manuscript. All authors read and approved the final manuscript.

\section{Ethics approval and consent to participate}

Not applicable.

\section{Patient consent for publication}

Not applicable.

\section{Competing interests}

The authors declare that they have no competing interests.

\section{References}

1. Bray F, Ferlay J, Soerjomataram I, Siegel RL, Torre LA and Jemal A: Global cancer statistics 2018: GLOBOCAN estimates of incidence and mortality worldwide for 36 cancers in 185 countries. CA Cancer J Clin 68: 394-424, 2018.

2. Brockstein BE: Management of recurrent head and neck cancer: Recent progress and future directions. Drugs 71: 1551-1559, 2011

3. de Ruijter AJM, van Gennip AH, Caron HN, Kemp S and van Kuilenburg ABP: Histone deacetylases (HDACs): Characterization of the classical HDAC family. Biochem J 370: 737-749, 2003.

4. Boyault C, Sadoul K, Pabion M and Khochbin S: HDAC6, at the crossroads between cytoskeleton and cell signaling by acetylation and ubiquitination. Oncogene 26: 5468-5476, 2007.

5. Gao YS, Hubbert CC, Lu J, Lee YS, Lee JY and Yao TP: Histone deacetylase 6 regulates growth factor-induced actin remodeling and endocytosis. Mol Cell Biol 27: 8637-8647, 2007.

6. Kawaguchi Y, Kovacs JJ, McLaurin A, Vance JM, Ito A and Yao TP: The deacetylase HDAC6 regulates aggresome formation and cell viability in response to misfolded protein stress. Cell 115 727-738, 2003

7. Scroggins BT, Robzyk K, Wang D, Marcu MG, Tsutsumi S, Beebe K, Cotter RJ, Felts S, Toft D, Karnitz L, et al: An acetylation site in the middle domain of Hsp90 regulates chaperone function. Mol Cell 25: 151-159, 2007

8. Li T, Zhang C, Hassan S, Liu X, Song F, Chen K, Zhang W and Yang J: Histone deacetylase 6 in cancer. J Hematol Oncol 11: 111, 2018.

9. Wang XX, Wan RZ and Liu ZP: Recent advances in the discovery of potent and selective HDAC6 inhibitors. Eur J Med Chem 143: 1406-1418, 2018.

10. Tabas I and Ron D: Integrating the mechanisms of apoptosis induced by endoplasmic reticulum stress. Nat Cell Biol 13: 184-190, 2011

11. Komatsu S, Moriya S, Che XF, Yokoyama T, Kohno N and Miyazawa K: Combined treatment with SAHA, bortezomib, and clarithromycin for concomitant targeting of aggresome formation and intracellular proteolytic pathways enhances ER stress-mediated cell death in breast cancer cells. Biochem Biophys Res Commun 437: 41-47, 2013.

12. Porter NJ, Mahendran A, Breslow R and Christianson DW: Unusual zinc-binding mode of HDAC6-selective hydroxamate inhibitors. Proc Natl Acad Sci USA 114: 13459-13464, 2017.

13. Mishima Y, Santo L, Eda H, Cirstea D, Nemani N, Yee AJ, O'Donnell E, Selig MK, Quayle SN, Arastu-Kapur S, et al: Ricolinostat (ACY-1215) induced inhibition of aggresome formation accelerates carfilzomib-induced multiple myeloma cell death. Br J Haematol 169: 423-434, 2015.

14. Komatsu S, Miyazawa K, Moriya S, Takase A, Naito M, Inazu M, Kohno N, Itoh M and Tomoda A: Clarithromycin enhances bortezomib-induced cytotoxicity via endoplasmic reticulum stress-mediated CHOP (GADD153) induction and autophagy in breast cancer cells. Int J Oncol 40: 1029-1039, 2012.
15. Moriya S, Che XF, Komatsu S, Abe A, Kawaguchi T, Gotoh A, Inazu M, Tomoda A and Miyazawa K: Macrolide antibiotics block autophagy flux and sensitize to bortezomib via endoplasmic reticulum stress-mediated CHOP induction in myeloma cells. Int J Oncol 42: 1541-1550, 2013.

16. Kazama H,Hiramoto M, Miyahara K, Takano N and Miyazawa K: Designing an effective drug combination for ER stress loading in cancer therapy using a real-time monitoring system. Biochem Biophys Res Commun 501: 286-292, 2018.

17. Takeda A, Takano N, Kokuba H, Hino H, Moriya S, Abe A, Hiramoto M, Tsukahara K and Miyazawa K: Macrolide antibiotics enhance the antitumor effect of lansoprazole resulting in lysosomal membrane permeabilization-associated cell death. Int J Oncol 57: 1280-1292, 2020.

18. Tanaka H, Hino H, Moriya S, Kazama H, Miyazaki M, Takano N, Hiramoto M, Tsukahara K and Miyazawa K: Comparison of autophagy inducibility in various tyrosine kinase inhibitors and their enhanced cytotoxicity via inhibition of autophagy in cancer cells in combined treatment with azithromycin. Biochem Biophys Rep 22: 100750, 2020.

19. Iwawaki T, Akai R, Kohno K and Miura M: A transgenic mouse model for monitoring endoplasmic reticulum stress. Nat Med 10: 98-102, 2003

20. Di Veroli GY, Fornari C, Wang D, Mollard S, Bramhall JL, Richards FM and Jodrell DI: Combenefit: An interactive platform for the analysis and visualization of drug combinations Bioinformatics 32: 2866-2868, 2016.

21. Amengual JE, Johannet P, Lombardo M, Zullo K, Hoehn D, Bhagat G, Scotto L, Jirau-Serrano X, Radeski D, Heinen J, et al: Dual targeting of protein degradation pathways with the selective HDAC6 inhibitor ACY-1215 and bortezomib is synergistic in lymphoma. Clin Cancer Res 21: 4663-4675, 2015.

22. Yin C and Li P: Growth suppression of glioma cells using HDAC6 inhibitor, tubacin. Open Med 13: 221-226, 2018.

23. Lohitesh K, Saini H, Srivastava A, Mukherjee S, Roy A and Chowdhury R: Autophagy inhibition potentiates SAHA-mediated apoptosis in glioblastoma cells by accumulation of damaged mitochondria. Oncol Rep 39: 2787-2796, 2018.

24. Kaizuka T, Morishita H, Hama Y, Tsukamoto S, Matsui T, Toyota Y, Kodama A, Ishihara T, Mizushima T and Mizushima N: An autophagic flux probe that releases an internal control. Mol Cell 64: 835-849, 2016.

25. Papadopoulos C, Kravic B and Meyer H: Repair or lysophagy: Dealing with damaged lysosomes. J Mol Biol 64: 835-849, 2020.

26. Kondo Y, Kanzawa T, Sawaya R and Kondo S: The role of autophagy in cancer development and response to therapy. Nat Rev Cancer 5: 726-734, 2005.

27. Bialik S, Dasari SK and Kimchi A: Autophagy-dependent cell death-where, how and why a cell eats itself to death. J Cell Sci 131: jcs215152, 2018.

28. Galluzzi L, Vitale I, Aaronson SA, Abrams JM, Adam D, Agostinis P, Alnemri ES, Altucci L, Amelio I, Andrews DW, et al: Molecular mechanisms of cell death: Recommendations of the Nomenclature Committee on Cell Death 2018. Cell Death Differ 25: 486-541, 2018.

29. Galluzzi L, Kepp O and Kroemer G: MLKL regulates necrotic plasma membrane permeabilization. Cell Res 24: 139-140,2014.

30. Huang D, Zheng X, Wang ZA, Chen X, He WT, Zhang Y, Xu JG, Zhao H, Shi W, Wang X, et al: The MLKL channel in necroptosis is an octamer formed by tetramers in a dyadic process. Mol Cell Biol 37: e00497-16, 2017.

31. Yan J: Interplay between HDAC6 and its interacting partners: Essential roles in the aggresome-autophagy pathway and neurodegenerative diseases. DNA Cell Biol 33: 567-580, 2014.

32. Morrow CS, Porter TJ, Xu N, Arndt ZP, Ako-Asare K, Heo HJ, Thompson EAN and Moore DL: Vimentin coordinates protein turnover at the aggresome during neural stem cell quiescence exit. Cell Stem Cell 26: 558-568.e559, 2020.

33. Johnston JA, Ward CL and Kopito RR: Aggresomes: A cellular response to misfolded proteins. J Cell Biol 143: 1883-1898, 1998.

34. Miyahara K, Kazama H, Kokuba H, Komatsu S, Hirota A, Takemura J, Hirasawa K, Moriya S, Abe A, Hiramoto M, et al: Targeting bortezomib-induced aggresome formation using vinorelbine enhances the cytotoxic effect along with ER stress loading in breast cancer cell lines. Int J Oncol 49: 1848-1858, 2016.

35. Marciniak SJ, Yun CY, Oyadomari S, Novoa I, Zhang Y, Jungreis R, Nagata K, Harding HP and Ron D: CHOP induces death by promoting protein synthesis and oxidation in the stressed endoplasmic reticulum. Genes Dev 18: 3066-77, 2004. 
36. Goodall ML, Fitzwalter BE, Zahedi S, Wu M, Rodriguez D, Mulcahy-Levy JM, Green DR, Morgan M, Cramer SD and Thorburn A: The autophagy machinery controls cell death switching between apoptosis and necroptosis. Dev Cell 37: 337-349, 2016.

37. Saito Y,Moriya S, KazamaH,HirasawaK,MiyaharaK, KokubaH, Hino H, Kikuchi H, Takano N, Hiramoto M, et al: Amino acid starvation culture condition sensitizes EGFR-expressing cancer cell lines to gefitinib-mediated cytotoxicity by inducing atypical necroptosis. Int J Oncol 52: 1165-1177, 2018.

38. Moriya S, Komatsu S, Yamasaki K, Kawai Y, Kokuba H, Hirota A, Che XF, Inazu M, Gotoh A, Hiramoto M and Miyazawa K: Targeting the integrated networks of aggresome formation, proteasome, and autophagy potentiates ER stress-mediated cell death in multiple myeloma cells. Int J Oncol 46: 474-486, 2015.

39. Tu BP and Weissman JS: Oxidative protein folding in eukaryotes: Mechanisms and consequences. J Cell Biol 164: 341-346, 2004.
40. Chakravarthi S, Jessop CE and Bulleid NJ: The role of glutathione in disulfide bond formation and endoplasmicreticulum-generated oxidative stress. EMBO Rep 7: 271-275, 2006.

41. Görlach A, Bertram K, Hudecova S and Krizanova O: Calcium and ROS: A mutual interplay. Redox Biol 6: 260-271, 2015.

42. Seervi M, Rani A, Sharma AK and Santhosh Kumar TR: ROS mediated ER stress induces Bax-Bak dependent and independent apoptosis in response to thioridazine. Biomed Pharmacother 106: 200-209, 2018.

43. Lin Y, Jiang M, Chen W, Zhao T and Wei Y: Cancer and ER stress: Mutual crosstalk between autophagy, oxidative stress and inflammatory response. Biomed Pharmacother 118: 109249, 2019. International (CC BY-NC-ND 4.0) License. 